OPEN ACCESS

Edited by:

Marco Ragusa,

University of Catania, Italy

Reviewed by:

Liu Shudong,

Agricultural University of Hebei, China Mohaddeseh Abouhosseini Tabari, Amol University of Special Modern

Technologies, Iran

Maria Grazia Morgese,

University of Foggia, Italy

*Correspondence: Aldo S. de Oliveira aldo.sena@ufsc.br

Leonardo L. G. Ferreira leonardo@ifsc.usp.br

Specialty section: This article was submitted to Experimental Pharmacology and Drug Discovery,

a section of the journa Frontiers in Pharmacology

Received: 03 October 2021 Accepted: 05 November 2021 Published: 23 November 2021

Citation:

de Oliveira AS, Llanes LC, Nunes RJ, Nucci-Martins C, de Souza AS,

Palomino-Salcedo $D L$

Dávila-Rodríguez MJ, Ferreira LLG

Santos ARS and Andricopulo $A D$

(2021) Antioxidant Activity, Molecular Docking, Quantum Studies and In Vivo

Antinociceptive Activity of

Sulfonamides Derived From Carvacrol.

Front. Pharmacol. 12:788850.

doi: 10.3389/fphar.2021.788850

\section{Antioxidant Activity, Molecular Docking, Quantum Studies and In Vivo Antinociceptive Activity of Sulfonamides Derived From Carvacrol}

\author{
Aldo S. de Oliveira ${ }^{1,2 *}$, Luana C. Llanes ${ }^{3}$, Ricardo J. Nunes ${ }^{4}$, Catharina Nucci-Martins ${ }^{5,6}$, \\ Anacleto S. de Souza ${ }^{2}$, David L. Palomino-Salcedo ${ }^{2}$, María J. Dávila-Rodríguez ${ }^{7}$, \\ Leonardo L. G. Ferreira ${ }^{2 *}$, Adair R. S. Santos ${ }^{5}$ and Adriano D. Andricopulo ${ }^{2}$ \\ ${ }^{1}$ Department of Exact Sciences and Education, Federal University of Santa Catarina-UFSC, Blumenau, Brazil, ${ }^{2}$ Laboratory of \\ Medicinal and Computational Chemistry, Institute of Physics of São Carlos, University of São Paulo-USP, São Carlos, Brazil, \\ ${ }^{3}$ Department of Chemistry and Biochemistry, University of California, Santa Barbara, Santa Barbara, CA, United States, \\ ${ }^{4}$ Departament of Chemistry, Federal University of Santa Catarina-UFSC, Florianópolis, Brazil, ${ }^{5}$ Department of Physiological \\ Sciences, Center of Biological Sciences, Federal University of Santa Catarina-UFSC, Florianópolis, Brazil, ${ }^{6}$ Department of \\ Structural and Functional Biology, Institute of Biology, University of Campinas-UNICAMP, Campinas, Brazil, ${ }^{7}$ Department of \\ Chemistry, Federal University of São Carlos-UFSCar, São Carlos, Brazil
}

The synthesis and antioxidant, antinociceptive and antiedematogenic activities of sulfonamides derived from carvacrol-a druglike natural product-are reported. The compounds showed promising antioxidant activity, and sulfonamide derived from morpholine (S1) demonstrated excellent antinociceptive and antiedematogenic activities, with no sedation or motor impairment. The mechanism that underlies the carvacrol and derived sulfonamides' relieving effects on pain has not yet been fully elucidated, however, this study shows that the antinociceptive activity can be partially mediated by the antagonism of glutamatergic signaling. Compound $\mathbf{S 1}$ presented promising efficacy and was predicted to have an appropriate medicinal chemistry profile. Thus, derivative $\mathbf{S} \mathbf{1}$ is an interesting starting point for the design of new leads for the treatment of pain and associated inflammation and prooxidative conditions.

Keywords: sulfonamides, pain, carvacrol, molecular modeling, antioxidant

\section{INTRODUCTION}

Pain is a major sorrowful condition that affects children, adolescents (Guindon et al., 2007; Schmidt et al., 2010) and adults (Loeser and Treede, 2008) in several pathologies, including cancer (Ling et al., 2012). Pain can impair daily activities, diminish life quality, and cause significant psychological conditions (Rowlingson, 2000).

Pain is a clinically meaningful sign for the detection and evaluation of many diseases. Its perception is complex, involving two distinct components, an emotional and a physiological or sensorial component, called nociception (Tominaga et al., 2003). Animal models used for the evaluation of antinociceptive activity involve several nociceptive responses generated by chemical, mechanical or thermal stimuli (Silva et al., 2013).

Despite advances in the pharmacokinetics and pharmacodynamics of analgesic agents, their high toxicity is a determinant of conflicting clinical results due to the need for drug associations and 


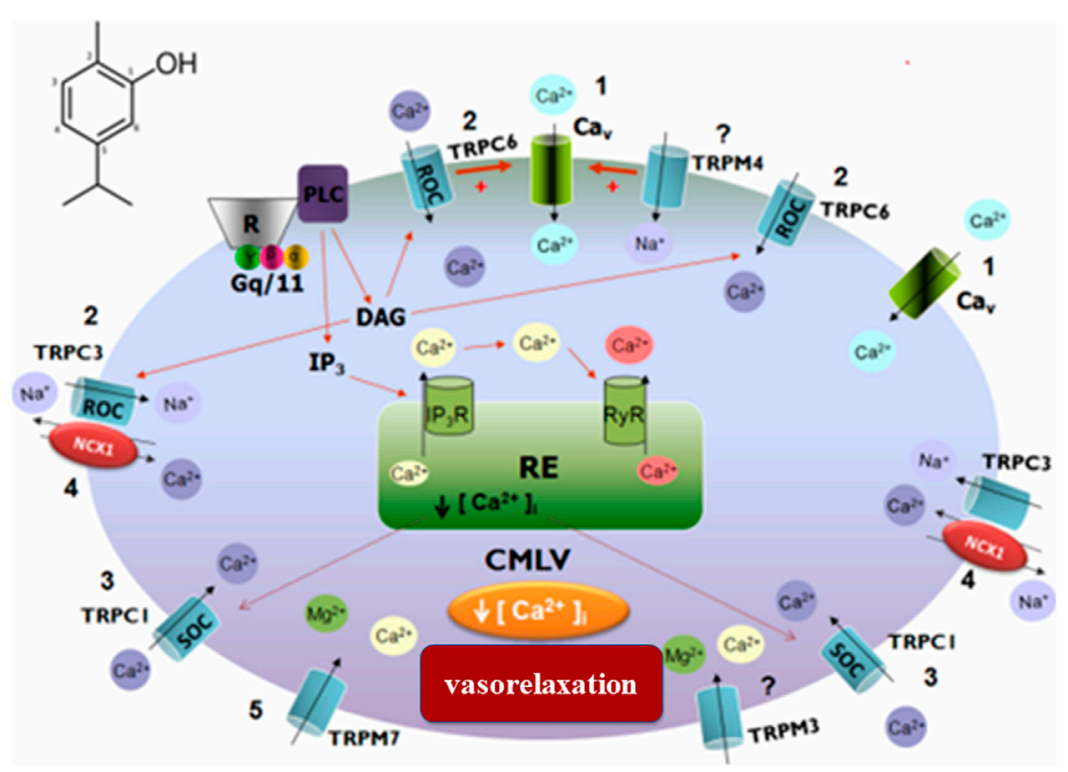

FIGURE 1 | Schematic representation of the probable signaling pathway of the vasorelaxant effect induced by carvacrol. 1) Blockage of calcium influx through the Cav; 2) Blockade of calcium influx through ROC and/or TRPC6; 3) Blockade of calcium influx through SOC and/or TRPC1; 4) Action on NCX1 by activation of TRPC3; 5) Inhibition of TRPM7

interactions, especially in chronic pain due to its bioplasticity, and association with clinical conditions of anxiety and depression that reduce the quality of life of patient.

Sound evidence indicates that amino acids, mainly glutamate, found in $\mathrm{C}$ and $\mathrm{A} \delta$ fibers, play a fundamental role in the transmission of pain, as they provoke post-synaptic depolarization and the propagation of nociceptive information (Verri et al., 2006). Besides, abnormal excitability caused by inflammation or injury usually results from increased expression or activation of receptors, which may be stimulated by glutamate, favoring the maintenance of the painful stimulus (Rao, 2009; Salvemini et al., 2011). Therefore, substances capable of causing selective changes in glutamatergic signaling may give rise to new analgesic and anti-inflammatory agents.

Upon inflammatory reactions, pro-inflammatory chemical messengers stimulate resident cells, recruit nociceptors and cells, and drive pain conduction (Manchope et al., 2016). Furthermore, augmented oxidative stress upon inflammation promotes nociception. For example, Reactive Nitrogen Species (RNS) and Reactive Oxygen Species (ROS) in a direct and indirect manner promote sensitization and activation of nociceptors (Maioli et al., 2015). The unbalance between oxidative and antioxidative agents in inflammatory reactions promotes oxidative stress (Biswas, 2016). Even though many analgesic agents can be used for the therapy of pain, research on novel drug candidates is needed considering that the current analgesics cause a broad diversity of adverse effects (Burgess and Williams, 2010).

Natural product structural motifs have been an invaluable source of new chemical matter for drug design and medicinal chemistry (Rodrigues et al., 2016). Recently, natural product research in the industry has decreased because of compatibility problems between natural-product extract collections and highthroughput screening platforms (Koehn and Carter, 2005). In this scenario, the monoterpene phenol 2-methyl-5-isopropyl-phenol, known as carvacrol, is a simple molecule with no stereogenic centers, with druglike properties and whose derivatives can be used for structure-activity relationship (SAR) studies. Along with the anti-inflammatory activity of carvacrol (Arigesavan and Sudhandiran, 2015), researchers have been interested in studying the analgesic action of this monoterpene.

Calcium and potassium channels are also directly related to the transmission of painful impulses since they are central for the release of neurotransmitters from nociceptor terminals. In this sense, studies demonstrate that carvacrol promotes a vasorelaxant response in upper mesenteric artery rings in rats, potentially because it inhibits the influx of calcium ions mediated by voltagesensitive calcium channels (Cav), as well as the receptor-operated channel (ROC) (Pires et al., 2015). Stock-actuated calcium channels (SOC) seem to be associated with classical TRP receptors (C6, C1, and TRPC) and also with melastatin TRP receptor channel inhibition (TRPM7) (Figure 1). The observed vasorelaxant activity may be involved in the hypotensive response detected in in vivo studies (Dantas et al., 2015).

Melo et al. (2010) demonstrated that doses of 12.5, 25, and $50 \mathrm{mg} / \mathrm{kg}$ of carvacrol, administered orally, have an anxiolytic effect and do not alter the locomotor activity of the animals. In a previous study, we demonstrated that some synthetic sulfonamides derived from carvacrol at a dose of $30 \mathrm{mg} / \mathrm{kg}$, intraperitoneal (ip), are able to reduce streptozotocin-induced Alzheimer's disease deficits, in addition to producing anxiolytic and antioxidant effects, without affecting locomotor activity of animals (de Souza et al., 2020). Also, it was confirmed that carvacrol, administered orally, at single doses of 50 and 


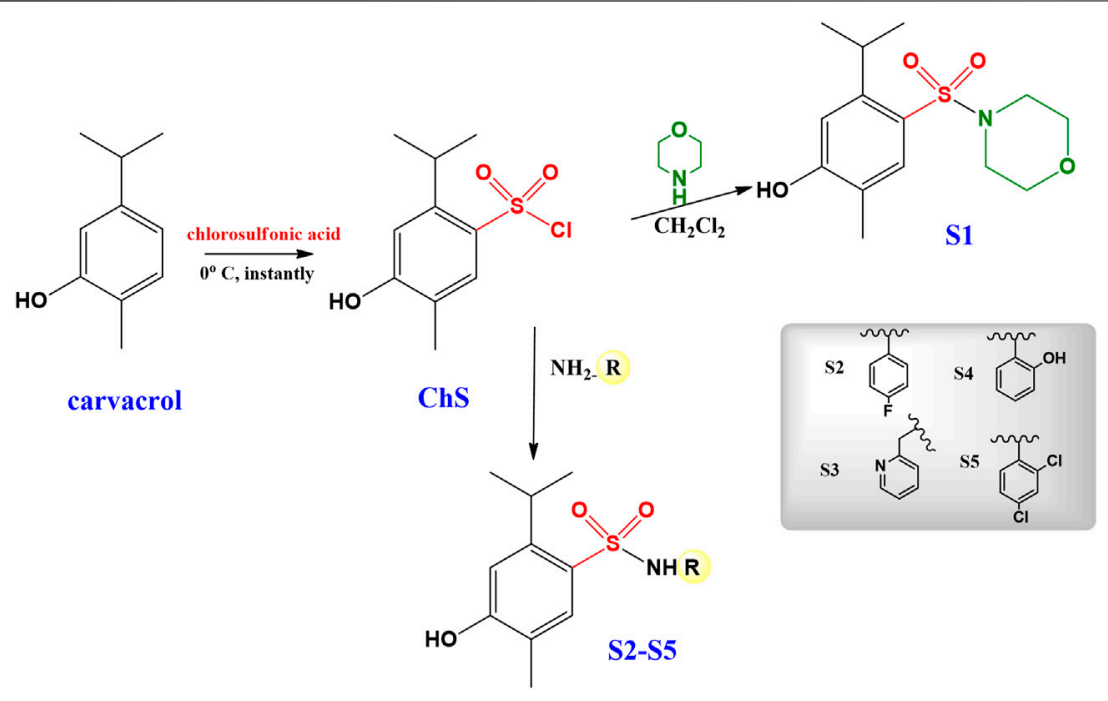

SCHEME 1 | Synthesis of the carvacrol-derived sulfonamides.

$100 \mathrm{mg} / \mathrm{kg}$, produces significant inhibition of nociception caused by chemical (formalin and acetic acid) and thermal stimulations (hot-plate test) (Cavalcante Melo et al., 2012). Furthermore, part of the mechanism by which carvacrol exerts its effects was demonstrated by Zotti et al. (2013). The authors found that carvacrol administered orally for seven consecutive days $(12.5 \mathrm{mg} / \mathrm{kg})$ was able to increase dopamine and serotonin levels in the prefrontal cortex and hippocampus. Following these findings, it has been demonstrated that carvacrol promotes antinociceptive effects by a mechanism that is independent on the activation of the opioid machinery and the L-arginine-nitric oxide (NO) pathway (Cavalcante Melo et al., 2012).

Sulfonamides derived from carvacrol have been investigated recently, for which antibacterial properties (Oliveira et al., 2020) and potential candidates for the development of drugs for the treatment of Alzheimer's disease have been reported (De Souza et al., 2020). As mentioned above and due to the analgesic and anti-inflammatory potential of carvacrol, in this research, the antinociceptive potential of these sulfonamides was investigated. Thus, this investigation is the first report to demonstrate the potential antioxidant activity of sulfonamides derived from carvacrol. Furthermore, this is the first report of sulfonamides derived from carvacrol, rationally designed to the effective control of pain via inhibition of the glutamatergic system. Additionally, molecular docking and quantum investigations were carried out to rationalize the in vitro and in vivo data.

Despite advances in the pharmacokinetics and pharmacodynamics of analgesic agents, their high toxicity is a determinant of conflicting clinical results due to the need for drug associations and interactions, especially in chronic pain due to its bioplasticity, and association with clinical conditions of anxiety and depression that reduce the quality of life of patient (Berman and Bausell, 2000; Jensen et al., 2001). Therefore, the development of new chemotherapeutic agents for pain treatment, which is the objective of this research, is extremely relevant in the context of public health worldwide.

\section{MATERIALS AND METHODS}

\section{Synthesis of Sulfonamides}

All the solvents used were analytically pure. The reagents 5isopropyl-2-methylphenol (carvacrol), chlorosulfonic acid, morpholine, 4-fluoroaniline, pyridin-2-yl methanamine, 2hydroxyaniline, 2,4-dichloroaniline were obtained from Sigma Aldrich.

The synthesis sulfonamides $\mathbf{S 1 - S 5}$, as already described in the literature (de Oliveira et al., 2016) was performed in two steps: firstly, the synthesis of 4-hydroxy-2-isopropyl-5methylbenzene-1-sulfonyl chloride (ChS) was performed, subsequently, the ChS was used in reactions with different amines (Scheme 1). ChS was obtained from the reaction of carvacrol to six equivalents of chlorosulfonic acid. The sulfonamides obtained in this study were prepared from ChS with two equivalents of amine added slowly. Reactions were followed by thin layer chromatography (TLC). All sulfonamides were purified by acid-base extraction and the compounds were duly characterized by spectroscopic and spectrometric techniques.

\section{Behavioral Tests Animal Models}

Animal care and in vivo procedures were carried out according to the ethical guides for the study in conscious animals of experimental pain (Zimmermann, 1983). The experiments were carried out after protocol approval from the Ethics Committee of the Federal University of Santa Catarina-UFSC (protocol PP00745). Male Swiss mice (25-35 g) were obtained 
from UFSC. Animals were maintained in a $12 \mathrm{~h}$ light $/ 12 \mathrm{~h}$ dark cycle (lights on at 6:00 a.m.) under a temperature of $22 \pm 2{ }^{\circ} \mathrm{C}$ with water and food ad libitum. At least $1 \mathrm{~h}$ before the tests, the animals were acclimatized to the laboratory conditions. The tests were executed from 8:00 a.m. to 12:00 a.m. The number of animals and noxious stimulation intensity were kept at the minimum needed to obtain consistent results.

\section{Drugs and Reagents}

The following substance was used: L-glutamic acid hydrochloride (Sigma-Aldrich, St. Louis, MO, United States). This formulation has a glutamate content of $\geq 99 \%$ measured by HPLC, according to the manufacturer's technical sheet. The carvacrol, used in this work, was obtained commercially in liquid form by SigmaAldrich, whose density is $0.976 \mathrm{~g} / \mathrm{ml}$ at $20^{\circ} \mathrm{C}$ (lit.), melting point $3-4^{\circ} \mathrm{C}$ (lit.) with a concentration of $98 \%$. Glutamate was solubilized in isotonic saline solution $(0.9 \% \mathrm{NaCl})$, and carvacrol and sulfonamides derived from carvacrol (S1-S5, Scheme 1) were dissolved in saline plus Tween 80 . Tween 80 did not exceed a $5 \%$ final concentration and did not show any activity by itself. Control groups for each delivery route were given isotonic saline with Tween 80 at $5 \%$.

\section{Glutamate-Induced Nociception}

To demonstrate the possible interplay between the carvacrol derivatives and the glutamatergic system, we evaluated whether the compounds would antagonize the glutamateinduced pain behavior of paw licking and biting. This glutamate-induced model of nociception was reported previously (Beirith et al., 2002; Meotti et al., 2010). A $20 \mu \mathrm{l}$ glutamate solution $(20 \mu \mathrm{mol} / \mathrm{paw}$, in saline, with $\mathrm{pH}$ adjusted to 7.4) was administered intraplantarly (i.pl.) in the ventral face of the right hind paw. After the administration of glutamate, the mice were monitored for $15 \mathrm{~min}$. Nociception was monitored by measuring with a chronometer the amount of time that mice spent licking and biting the injected paw. The mice were given vehicle intragastrically (i.g.) $(10 \mathrm{ml} / \mathrm{kg})$ or carvacrol derivatives $(0.0003,0.003$, and $0.03 \mathrm{mg} / \mathrm{kg}) \quad 1 \mathrm{~h}$ before glutamate administration.

Additionally, the thickness of the animal paw was measured with a digital micrometer $(0-25 \mathrm{~mm})$ before and after the nociceptive response induced by glutamate (i.pl.) to evaluate the paw edema. The difference in thickness $(\mathrm{mm})$ of the hind paw, immediately before and after the test of glutamate, was considered as an index of edema.

\section{Evaluation of Locomotor Activity}

The open-field test is widely used to assess spontaneous locomotor activity in animals to exclude possible nonspecific effects of a drug on the central nervous system (CNS), causing sedation or motor dysfunction. This is an important measure to check for possible false positives in pain studies, as these parameters can be easily confused with an analgesic effect of the evaluated drug and cause research bias. Thus, to examine the activity of the carvacrol derivatives on spontaneous locomotion, the open-field test was performed as described above (Nucci-Martins et al., 2016; de Souza et al., 2020). The open-field test device was a wooden box
$(40 \times 60 \times 50 \mathrm{~cm})$. The floor was split into 12 equal squares, and the number of squares that the animal covered with all paws in a 6 min session was registered. Mice were given the compounds (i.g., 0.0003, 0.003 , and $0.03 \mathrm{mg} / \mathrm{kg}$ ) or vehicle (i.g., $10 \mathrm{ml} / \mathrm{kg}$ ) $1 \mathrm{~h}$ before the test. Healthy mice that were not submitted to painful stimuli were used for the assessment of locomotor activity in the open-field experiment.

\section{Statistical Analyses}

Results are reported as average values \pm standard deviation (SD) with the exception of $\mathrm{ID}_{50}$ and $\mathrm{EC}_{50}$ values, which were calculated from single experiments using nonlinear regression implemented in GraphPad 7.0 (GraphPad software, San Diego, CA, United States). The glutamate test with paw edema measurement and the open-field test showed a normal data distribution in line with the Shapiro-Wilk threshold ( $p=0.05$ ) and, thus, were submitted to one-way ANOVA analysis and to Dunnett test for multiple analyses. Only $p$-values below 0.05 were taken as significant $(p<0.05)$.

\section{Antioxidant Assays}

\section{Scavenging Assay-Nitric Oxide}

NO scavenging assay was performed using the method reported by Sens et al. (2018). In this assay, sodium nitroprusside generates NO radicals $(\mathrm{NO} \bullet)$ which react with oxygen to generate nitrite ions. The production of the nitrite ions is then determined with the Griess reagent ( $1 \%$ sulfanilamide, $2 \% \mathrm{H}_{3} \mathrm{PO}_{4}$ and $0.1 \%$ naphthylethylenediamine dihydrochloride). NO scavenging activity was measured by adding $1.5 \mathrm{ml}$ phosphate buffer saline (0.2 M, pH 7.4) and $1 \mathrm{ml}$ sodium nitroprusside $(10 \mathrm{mM})$ to several concentrations of the test compounds $(25,50,75$, and $100 \mathrm{mg} \mathrm{ml}^{-1}$ ) and incubating the reaction mixture for $150 \mathrm{~min}$ $\left(25^{\circ} \mathrm{C}\right)$. Next, $1 \mathrm{ml}$ of Griess reagent was added to $1 \mathrm{ml}$ of the reaction solution. A wavelength of $546 \mathrm{~nm}$ was set to measure absorbance (A), and the results of antioxidant assays were expressed as $\mathrm{EC}_{50}$.

\section{Scavenging Assay-Hydrogen Peroxide}

The $\mathrm{H}_{2} \mathrm{O}_{2}$ scavenging activity showed by the compounds was measured spectrophotometrically using a method reported previously (Sens et al., 2018). A $40 \mathrm{mM} \mathrm{H}_{2} \mathrm{O}_{2}$ solution was made in phosphate buffer ( $\mathrm{pH} 7.4$ ). 25, 50, 75, and $100 \mathrm{mg} \mathrm{ml}^{-1}$ test compound solutions in phosphate buffer $(3.4 \mathrm{ml})$ were added to the $\mathrm{H}_{2} \mathrm{O}_{2}$ solution $(0.6 \mathrm{ml})$. Absorbance was monitored at a wavelength of $230 \mathrm{~nm}$. The percentage of $\mathrm{H}_{2} \mathrm{O}_{2}$ scavenging was calculated, and the results were expressed as $\mathrm{EC}_{50}$.

\section{Computational Studies}

\section{Small-Molecule Modeling and Preparation}

All compounds were built in the Avogadro program (Hanwell et al., 2012). The structures of the compounds were optimized at $\mathrm{pH} 7.4$ to simulate the conditions found experimentally. Next, the compounds were minimized with the MMFF94s force field (Halgren, 1996) and the conjugate gradient method.

\section{Density Functional Theory}

All energy values of the lowest unoccupied molecular orbitals (LUMO) and highest occupied molecular orbitals (HOMO) were computed by the GAMESS (General Atomic and Molecular 
Electronic Structure System) software (Schmidt et al., 1993). In the calculation of simple energy, the Becke's three-parameter hybrid functional, the Lee-Yang-Parr correlation (B3LYP) functional (Nageswari et al., 2018) and the 6-31G(d, p) basis set were used in these molecular systems in gas phase, considering the neutral and singlet structures. The computation was run considering the Slater exchange potential correlation and the grid methodology. The Hückel method (Hückel, 1931) generated an initial estimate of molecular orbitals and electronic density. Consequently, the self-consistent field (SCF) convergence was attributed by the restricted Hartree-Fock (RHF) method (Schmidt et al., 1993), which was limited to 30 iteration cycles. LUMO and HOMO potentials were compared with the experimental results of $\mathrm{NO}\left(\mathrm{EC}_{50}{ }^{\mathrm{NO}}\right)$ and peroxide $\left(\mathrm{EC}_{50}{ }^{\mathrm{H} 2 \mathrm{O} 2}\right)$ elimination activities. Finally, HOMO-biological activity $\left(\mathrm{EC}_{50}{ }^{\mathrm{NO}}\right.$ and $\mathrm{EC}_{50}{ }^{\mathrm{H} 2 \mathrm{O} 2}$ ) linear regression models were developed.

\section{Molecular Docking}

The PDB (Berman et al., 2002) was searched for structures of Rattus norvegicus bound to antagonist corresponding to the UniProt Gene Names Grin1 and Grin2A-D (NMDA receptors; 23 structures found); Gria1-4 (AMPA receptors; 16 structures found); Grik1-5 (Kainate receptors; 20 structures found); Grm1 and Grm5 (mGluR Group I receptors; 1 structure found); Grm2-3 (mGluR Group II receptors; no structures found) and Grm4-8 (mGluR Group III receptors; no structures found). When more than one structure was available, a direct comparison of the binding sites was performed to evaluate their plasticity and select the smallest subset of structures capable of representing it. For each subset, ensemble docking calculations were performed. After identifying the structure of each receptor with a higher affinity for the compounds, docking simulations were performed individually. The structural data of the heme domain of rat neuronal NO synthase bound to 6-(3fluoro-5-(3-(methylamino)prop-1-yn-1-yl)phenethyl)-4methylpyridin-2-amine (PDB 6NGJ) was additionally used.

In all docking calculations, performed with GOLD v.5.6.1 and the ChemPLP (Korb et al., 2009) scoring function, the receptors were kept rigid, and the ligands were treated with full flexibility. The receptors were prepared using GOLD, and structural water molecules were not considered. The atoms up to a distance of $8 \AA$ from the crystallographic ligands in both the ensemble and individual docking simulations were considered to define the binding sites. PyMOL v.1.8 (Schrödinger, New York, NY) was used to create the receptor-ligand figures.

\section{Molecular Properties and Pharmacokinetics}

Molinspiration Chemoinformatics was used for calculating Octanol-Water Partition Coefficient (milogP), number of atoms (natoms), Topological Polar Surface Area (TPSA), molecular weight (MW), hydrogen bond donors (HBD) and hydrogen bond acceptors (HBA), rotatable bonds (NRB), Molecular Volume, and Lipinski RO5 violations.

The SwissADME tool (http://www.swissadme.ch) was employed for the generation of the Bioavailability Radar, and assess lipophilicity, druglikeness, medicinal chemistry and pharmacokinetics parameters.
TABLE 1 | Antioxidant activity of sulfonamides derived from carvacrol.

\begin{tabular}{lcc} 
Compound & $\begin{array}{c}\text { NO scavenging activity } \\
\mathbf{E C}_{\mathbf{5 0}}(\boldsymbol{\mu} \mathbf{M})\end{array}$ & $\begin{array}{c}\mathbf{H}_{\mathbf{2}} \mathbf{O}_{\mathbf{2}} \text { scavenging activity } \\
\mathbf{E C}_{\mathbf{5 0}}(\boldsymbol{\mu M} \mathbf{M})\end{array}$ \\
\hline S1 & $12.25 \pm 0.12$ & $13.13 \pm 0.11$ \\
S2 & $18.11 \pm 0.14$ & $20.16 \pm 0.17$ \\
S3 & $12.14 \pm 0.28$ & $13.85 \pm 0.33$ \\
S4 & $18.76 \pm 0.22$ & $20.28 \pm 0.14$ \\
S5 & $12.04 \pm 0.11$ & $13.12 \pm 0.18$ \\
Ascorbic acid & $14.72 \pm 0.23$ & $16.3 \pm 0.26$
\end{tabular}

\section{RESULTS}

The synthetic procedures for the sulfonamides S1-S5 (Scheme 1), following a recently reported methodology (de Oliveira et al., 2016), were performed in good yields (85-95\%).

\section{Antioxidant Activity}

The antioxidant activity of the sulfonamides derived from carvacrol (Table 1) was analyzed by the $\mathrm{NO}$ and $\mathrm{H}_{2} \mathrm{O}_{2}$ scavenging activity assays.

\section{Quantum Studies}

The electronic properties were directly correlated with the antioxidant activity of the molecules. The $\mathrm{E}_{\mathrm{HOMO}}$ and $\mathrm{E}_{\mathrm{LUMO}}$ indicate the molecule's ability to donate and receive electron density, respectively. The difference between the two energy levels is termed the band gap and gives an estimate of the reactivity of a molecule. The distance between the HOMO and LUMO energy levels is inversely proportional to the reactivity the compound. The HOMO and LUMO potentials and band gap of the carvacrol derivatives are shown in Figure 2.

Figure 3 shows the correlation between HOMO energy and experimental $\mathrm{EC}_{50}{ }^{\mathrm{NO}}$ and $\mathrm{EC}_{50}{ }^{\mathrm{H} 2 \mathrm{O}}$. The correlation coefficients $r^{2}$ and Person's coefficient $(r)$ of the $\mathrm{EC}_{50}{ }^{\mathrm{NO}}$ versus $\mathrm{E}^{\mathrm{HOMO}}$ were 0.87 and 0.93 , respectively. For $\mathrm{EC}_{50}{ }^{\mathrm{H} 2 \mathrm{O} 2}$ versus $\mathrm{E}^{\mathrm{HOMO}}, r^{2}$ and $r$ were 0.88 and 0.94 , respectively. The angular coefficient values of the equations $\mathrm{EC}_{50} \mathrm{NO}=\mathrm{EC}_{50} \mathrm{NO}\left(\mathrm{E}^{\mathrm{HOMO}}\right)$ and $\mathrm{EC}_{50}{ }^{\mathrm{H} 2 \mathrm{O} 2}=\mathrm{EC}_{50}{ }^{\mathrm{H} 2 \mathrm{O} 2}\left(\mathrm{E}^{\mathrm{HOMO}}\right)$ were, respectively, $10.28 \pm 2.03$ and $11.30 \pm 2.10 \mu \mathrm{mol}$. $(\mathrm{L} . \mathrm{eV})^{-1}$. In addition, the linear coefficients were $87.90 \pm 14.49$ and $96.63 \pm 14.96 \mu \mathrm{mol}$. $(\mathrm{L} . \mathrm{eV})^{-1}$, respectively. From these equations, the minimal values of $\mathrm{E}^{\mathrm{HOMO}}$ (i.e., $\mathrm{EC}_{50}{ }^{\mathrm{NO}}=\mathrm{EC}_{50}{ }^{\mathrm{H} 2 \mathrm{O} 2}=0$ ) can find the maximal activity. Thus, with the HOMO energy tending to $-8.55 \mathrm{eV}$ for both equations, the maximal elimination of $\mathrm{NO}$ and $\mathrm{H}_{2} \mathrm{O}_{2}$ is reached for both experiments.

\section{Antinociceptive Activity}

For a better understanding of the antinociceptive effect of sulfonamides derived from carvacrol (S1-S5), we used the model of glutamate-induced (i.pl.) nociception. This method allowed us to investigate the possible interaction of peripheral antinociceptive action of the analyzed compounds with the glutamatergic system. The results are shown in Figure 4.

Figure 5 shows the results of treatment with carvacrol and its derivatives on paw edema induced by glutamate (i.pl.). Our results show that only $\mathbf{S 1}$ and $\mathbf{S 5}$ were able to significantly reduce edema. 

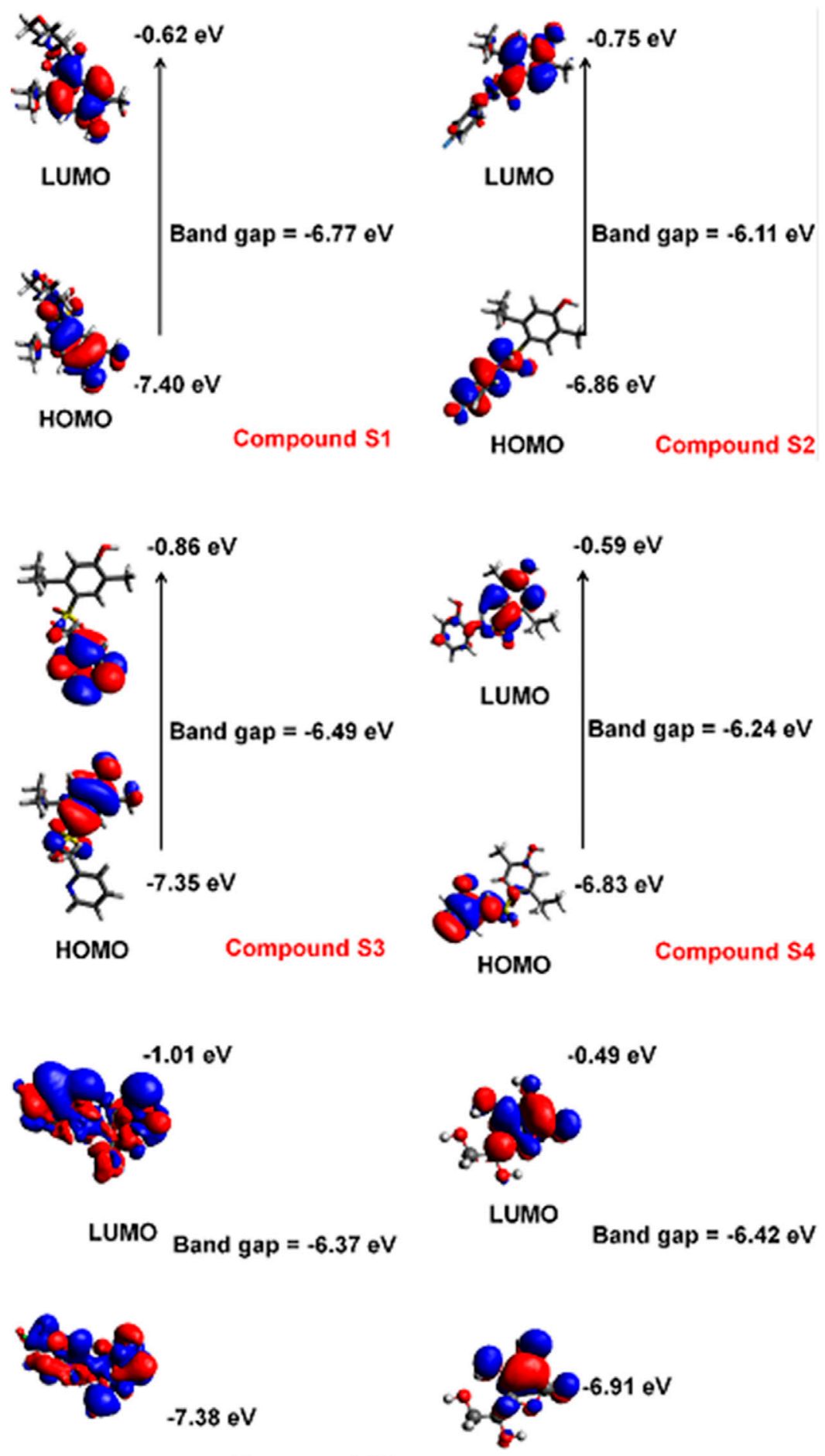

HOMO

Compound S5

HOMO

Ascorbic acid

FIGURE 2 | HOMO and LUMO potentials of the carvacrol derivatives estimated by the B3LYP method and 6-31G(d,p) basis set.

However, $\mathbf{S 1}$ inhibited edema more effectively and dose-dependently. The percent inhibition values were: $36 \pm 10 \%, 47 \pm 6 \%$, and $73 \pm 12 \%$ for $\mathrm{S} 1$ at $0.0003,0.003$ and $0.03 \mathrm{mg} / \mathrm{kg}$ i.g., respectively; $19 \pm 9 \%$, $33 \pm 6 \%$, and $28 \pm 7 \%$ for $\mathbf{S} 5$ at $0.0003,0.003$, and $0.03 \mathrm{mg} / \mathrm{kg}$ i.g., respectively. The value of $\mathrm{ID}_{50}$ for compound $\mathrm{S} 1$ was 0.002 $(0.0009-0.005) \mathrm{mg} / \mathrm{kg}$. Furthermore, the calculated values for the $\mathrm{ID}_{50}$ antiedematogenic effect of $\mathbf{S 1}(0.002 \mathrm{mg} / \mathrm{kg})$ agree with the dose found in the glutamate test, showing homogeneity of the data in this 

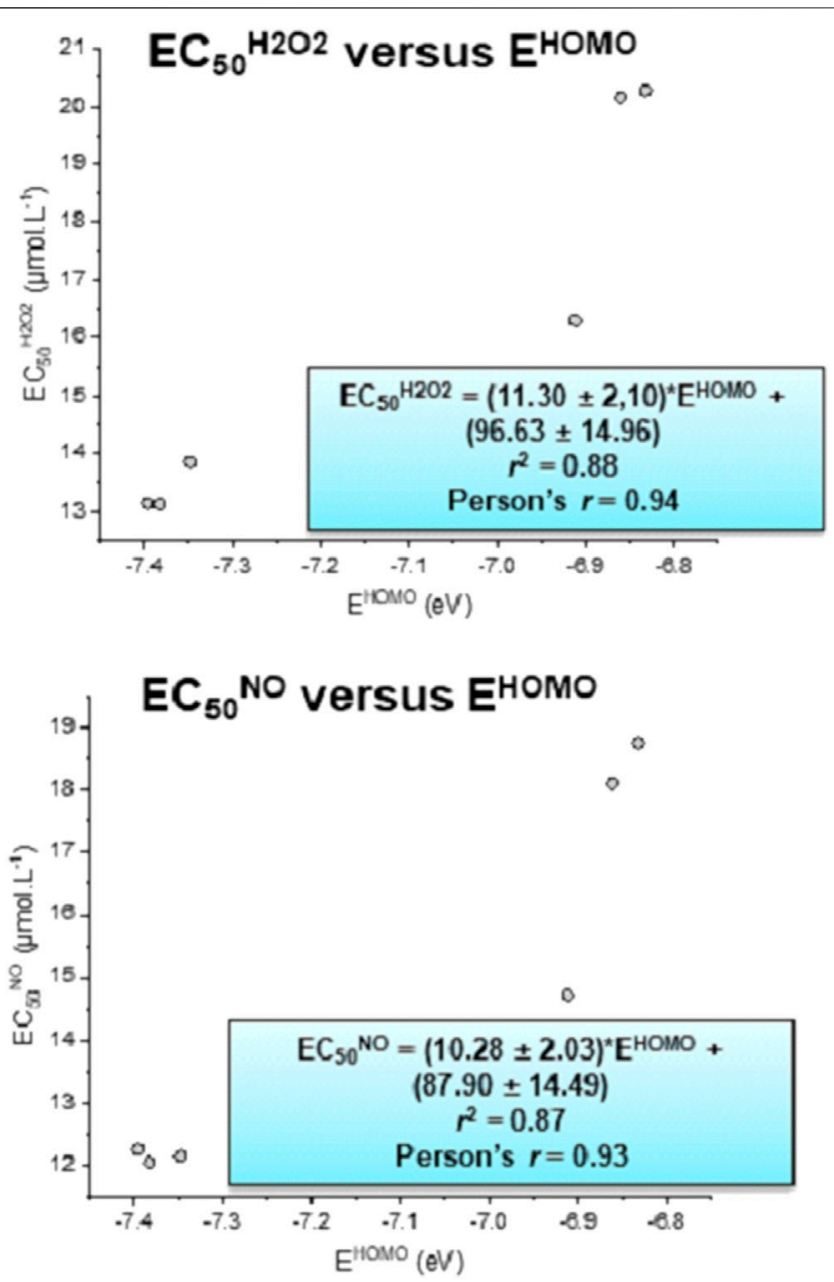

FIGURE 3 | HOMO energy ( $\mathrm{E}^{\mathrm{HOMO}}$ ) correlated with $\mathrm{EC}_{50}{ }^{\mathrm{NO}}$ and $\mathrm{EC}_{50}{ }^{\mathrm{H} 2 \mathrm{O} 2}$.

group. Thus, we suggest that $\mathbf{S 1}$ may be an interesting target for the reduction of edema in inflammatory conditions.

Figure 6 shows that intragastric administration of carvacrol and compounds S1, S2, S3, S4, and S5 at doses ranging from 0.0003 to $0.03 \mathrm{mg} / \mathrm{kg}$ had no effect on the locomotion of animals in comparison with the animals in the control group, suggesting that the compounds do not induce impairment of motor function in the animals. These results exclude the possibility that the antinociceptive action of carvacrol and its derivatives is nonspecifically associated with activity on the peripheral or central levels of locomotion control, such as sedation or motor dysfunction.

\section{Molecular Docking}

As previously shown (Fundytus, 2001), the administration of glutamate receptor (GluR) antagonists has an analgesic effect on peripheral pain. To assess whether the mechanism of action of S1-S5 is likely to involve these receptors, molecular docking simulations were performed over different GluR structures of Rattus norvegicus bound to antagonists (Supplementary Table S1).
For the predicted binding modes of S1-S5, the main interactions involving the common scaffold are hydrogen bonds with Gln405, Arg523, Thr518 and Ser572 and a displaced $\pi$-stacking interaction with Phe484. Of these, the interactions with Arg523, Thr518, and Phe484 are also observed for the crystallographic antagonist TK40 (Ravn et al., 2013). The main interactions observed for carvacrol are only hydrogen bonds with Pro516 and Thr518 and the displaced $\pi$-stacking interaction with Phe484 (Figure 7). For the different $R$ groups, mainly van der Waals interactions were established. Only for the $R$ groups of $\mathbf{S 3}$ and $\mathbf{S 5},-\mathrm{CH} \cdots \pi$ interactions with Leu538 and Ser572, respectively, were observed. Among all five molecules, S4 established the lowest number of contacts. The scores of each analyzed pose are presented in Supplementary Table S2.

The three levels of perception of pain-the cerebral (Dickenson, 1995), spinal and peripheral (Gordh et al., 1995) - appear to be affected by NO. This compound is an essential regulator of various immune and inflammatory functions (Moncada et al., 1991). In this work, we investigated, besides the NO scavenging activity, the possible intermolecular interactions between the sulfonamides and $\mathrm{NO}$ synthase. First, to validate the molecular docking protocol, redocking analysis (Figure 8) of 5,6,7,8-tetrahydrobiopterin (the crystallographic ligand, PDB ID 6NGJ) (Do et al., 2019) was carried out with GOLD. The ligand occupied the same interaction site in molecular docking when compared to the crystallographic structure, with emphasis on hydrogen bond interactions with Ser334, Val677, and Arg 596 and a $\pi$ interaction with Trp678.

The molecular docking results agree with the results obtained in the in vitro (NO scavenging activity) and in vivo tests. All compounds showed an inhibitory profile against NO synthase, except $\mathbf{S 4}$, which was not effective in all performed assays. The two most active compounds, $\mathbf{S} \mathbf{1}$ and S5 presented lower $\mathrm{ID}_{50}$ values and higher values for the scoring function, which demonstrate the high correlation between the in vivo and in silico results. The higher activity of these compounds was probably due to $\pi$ stacking interactions and a hydrogen bond between compounds S1 and S5 and Trp 678 (Figure 9), which were also observed for the co-crystallized ligand, but was not found for the other sulfonamides. The scores of each analyzed pose are presented in Supplementary Table S3.

\section{Molecular Properties}

Physicochemical and topological parameters of compounds S1-S5 were estimated to evaluate their pharmacokinetics profile. The octanol-water partition coefficient (miLogP), topological polar surface area (TPSA), molecular weight (MW), number of atoms, hydrogen-bond acceptors (HBA) and hydrogen-bond donors (HBD), number of rotatable bonds (NRB), Lipinski RO5 violations, and molecular volume are presented in Table 2. The silico-derived descriptor values were compared with the solubility and permeability filters for drug candidates reported by Lipinski (Barret, 2018), Oprea and Veber (Veber et al., 2002). 

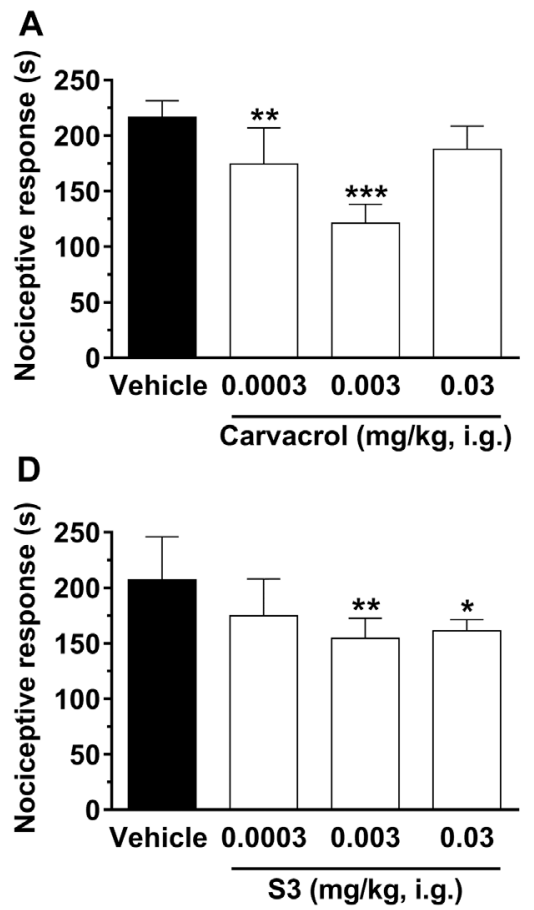
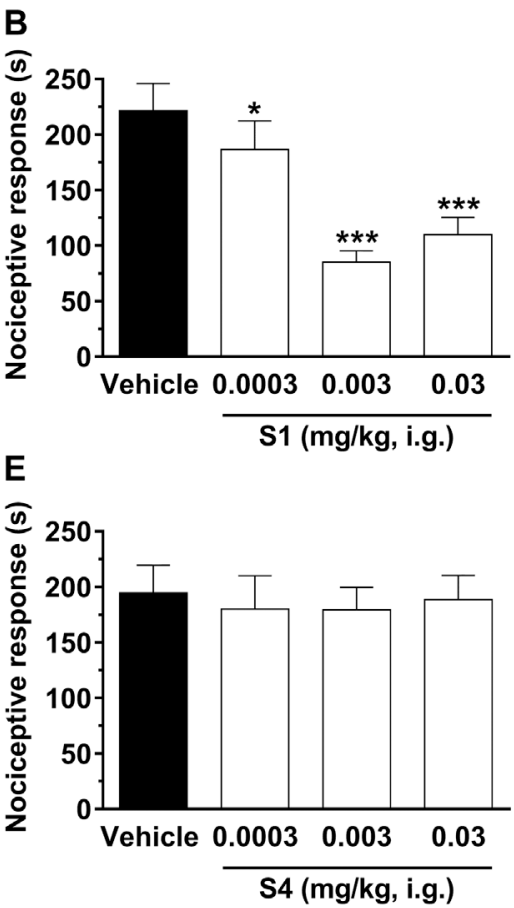

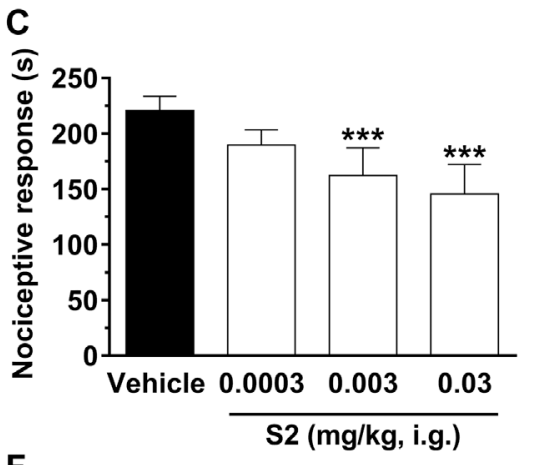

$\mathbf{F}$

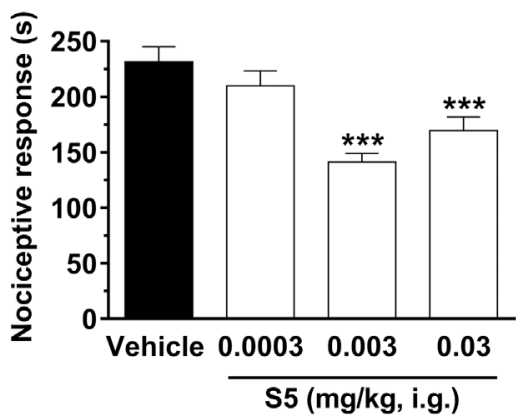

FIGURE 4 | Effect of compounds on nociception induced by glutamate (i.pl.) in mice. The pain behavior, translated by the nociceptive response of licking/biting hind paws induced by glutamate (i.pl.), was evaluated 1 hour after treatment with carvacrol (A), S1 (B), S2 (C), S3 (D), S4 (E) and S5 (F) at doses ranging from $0.0003,0.003$, and $0.03 \mathrm{mg} / \mathrm{kg}$, i.g., (open bars) or vehicle/control (closed bar). Each bar denotes the average response for 6-8 animals, and the vertical lines represent the SEM (standard error of mean). Asterisks $\left(^{*}\right)$ indicate the significance in comparison with the control group animals $\left({ }^{*} p<0.05\right.$, ${ }^{* *} p<0.01$, and $\left.{ }^{* \star *} p<0.001\right)$. One-way ANOVA and Dunnett test for multiple comparisons were used to determine the statistical significance.

The Swiss ADME web tool used to calculate the parameters is available at http://www.swissadme.ch and allows straightforward submission and analysis. It allows different input methods, multi-molecule computation, and offers the possibility to view and save results for each molecule, in addition to an interactive and intuitive visualization tool. To study the ADME parameters of the most active sulfonamide in the in vitro and phenotypic tests (S1), the Bioavailability Radar (Figure 10), lipophilicity, drug likeness (Figure 11), medicinal chemistry and pharmacokinetics (Figure 12) parameters were analyzed.

The Bioavailability Radar (Figure 10) provides a graphical output for the drug-likeness of a compound. The central shaded surface is the optimal domain for lipophilicity (XLOGP3 from -0.7 to +5.0 ), size (MW from 150 to $500 \mathrm{~g} / \mathrm{mol}$ ), polarity (TPSA from 20 to $130 \AA^{2}$ ), aqueous solubility ( $\log S \leq 6$ ), saturation (fraction of sp3 carbons $\geq 0.25$ ), and flexibility (rotatable bonds $\leq$ 9). Compound $\mathbf{S 1}$ falls within the optimal range for all parameters.

In addition, $\mathbf{S} \mathbf{1}$ has a good medicinal chemistry and synthetic accessibility profile, which is very important in obtaining a drug that can be commercially distributed at a more affordable price. Moreover, $\mathbf{S} \mathbf{1}$ has high gastrointestinal absorption (GI) and blood-brain barrier permeability (Figure 12).

\section{DISCUSSION}

\section{Antioxidant Activity}

The evaluation of the antioxidant activity of a series of compounds should be performed in more than one experiment, allowing for the reliability of the results (Sens et al., 2018). Diverse in vitro antioxidant assays have been published. Herein, the antioxidant ability of derivatives S1-S5 was determined in two in vitro tests. Subsequently, the results of these tests were correlated with the findings from the HOMO and LUMO studies.

Compounds S1, S3, and S5 were more active than ascorbic acid (AA), which was used as the reference compound. Compound S5 showed the highest activity, and S4 demonstrated to be the least active. A linear correlation was found between both experimental results $\left(\mathrm{EC}_{50}{ }^{\mathrm{H} 2 \mathrm{O} 2}=1.085 \mathrm{EC}_{50}{ }^{\mathrm{NO}}+0.2250 ; r^{2}=0.99\right)$.

NO plays a critical part in the control of multiple physiological responses. Also, the $\mathrm{NO}$ cascade is associated with many conditions, including Alzheimer's disease (Di Meo et al., 2016). $\mathrm{H}_{2} \mathrm{O}_{2}$ readily decomposes into water and oxygen, resulting in the production of hydroxyl radicals $(\mathrm{OH} \bullet)$, lipid peroxidation and DNA injury, which makes it a target for research of new compounds with antioxidant properties (Phaniendra et al., 2015). 

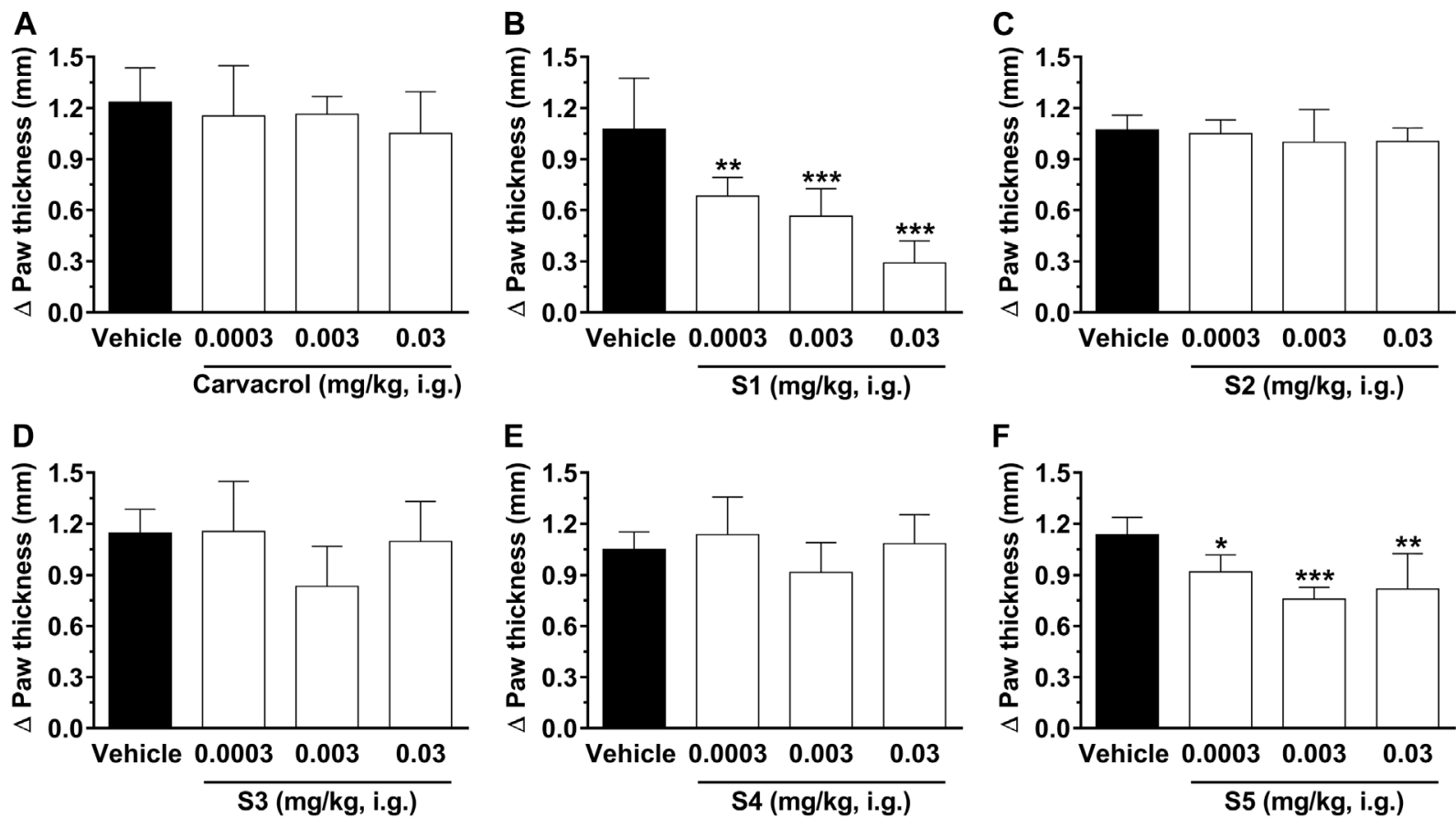

FIGURE 5 | Effect of compounds on paw edema in rats induced by glutamate (i.pl.). The edema was evaluated 1 hour after treatment with carvacrol (A), $\mathbf{S 1}$ (B), $\mathbf{S 2}$ (C), S3 (D), $\mathbf{S 4}$ (E) and S5 (F) at doses ranging from 0.0003, 0.003, and 0.03 mg/kg, i.g., (open bars) or vehicle/control (closed bar). The animal paw thickness difference was observed before and after the glutamate test. Each bar denotes the average response for $6-8$ animals, and the vertical lines represent SD. Asterisks (*) indicate the significance in comparison with the control group animals $\left({ }^{*} p<0.05,{ }^{\star \star} p<0.01\right.$, and $\left.{ }^{* \star *} p<0.001\right)$. One-way ANOVA and Dunnett test for multiple comparisons were used to determine the statistical significance.

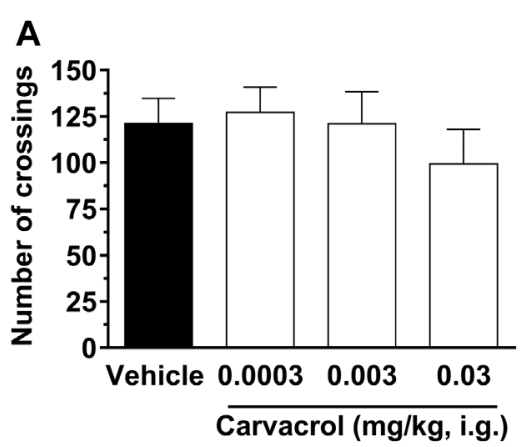

D

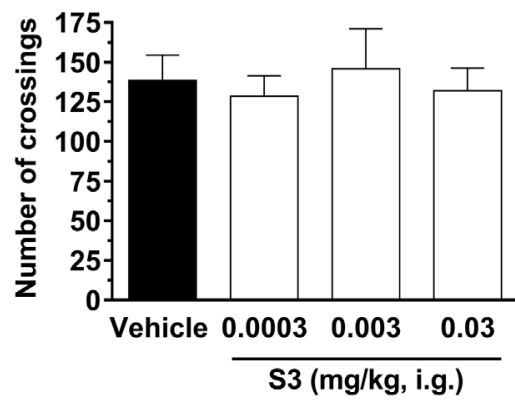

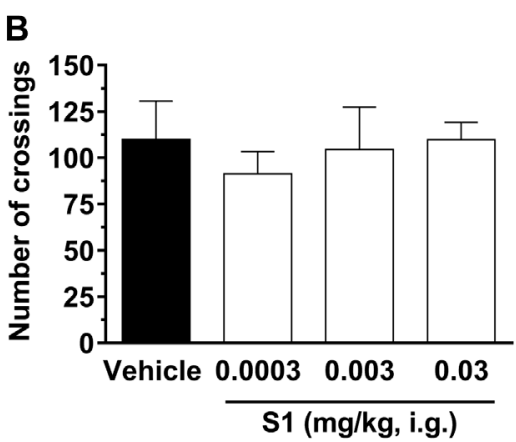

$E$

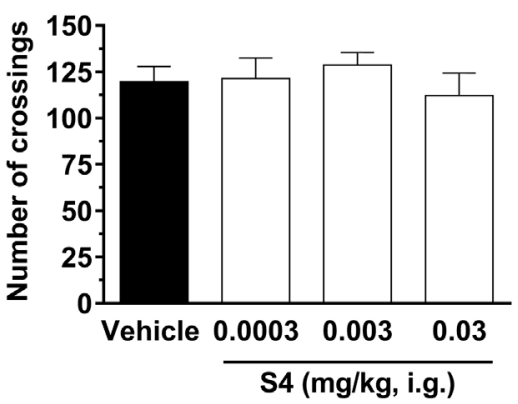

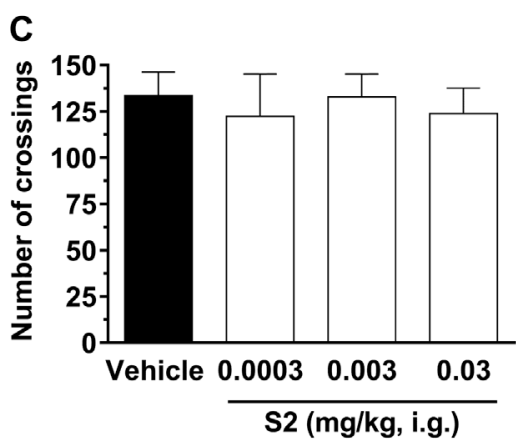

$F$

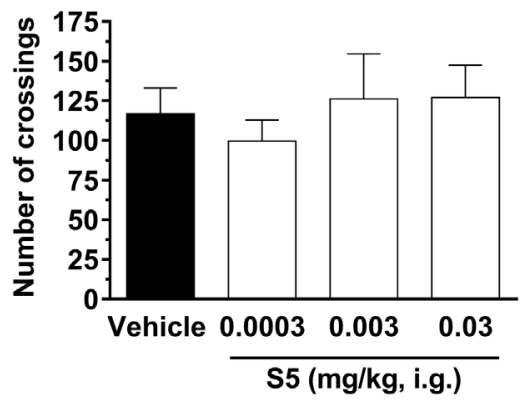

FIGURE 6 | Effect of compounds on the spontaneous locomotion of animals. The crossings were evaluated $1 \mathrm{~h}$ after treatment with carvacrol (A), $\mathbf{S 1}$ (B), $\mathbf{S 2}$ (C), S3 (D), S4 (E) and S5 (F) at doses ranging from 0.0003, 0.003, and 0.03 mg/kg, i.g., (open bars) or vehicle/control (closed bar). Each bar denotes the average values for 6-8 animals, and the vertical lines represent SD. One-way ANOVA and Dunnett test for multiple comparisons were used to determine the statistical significance. 


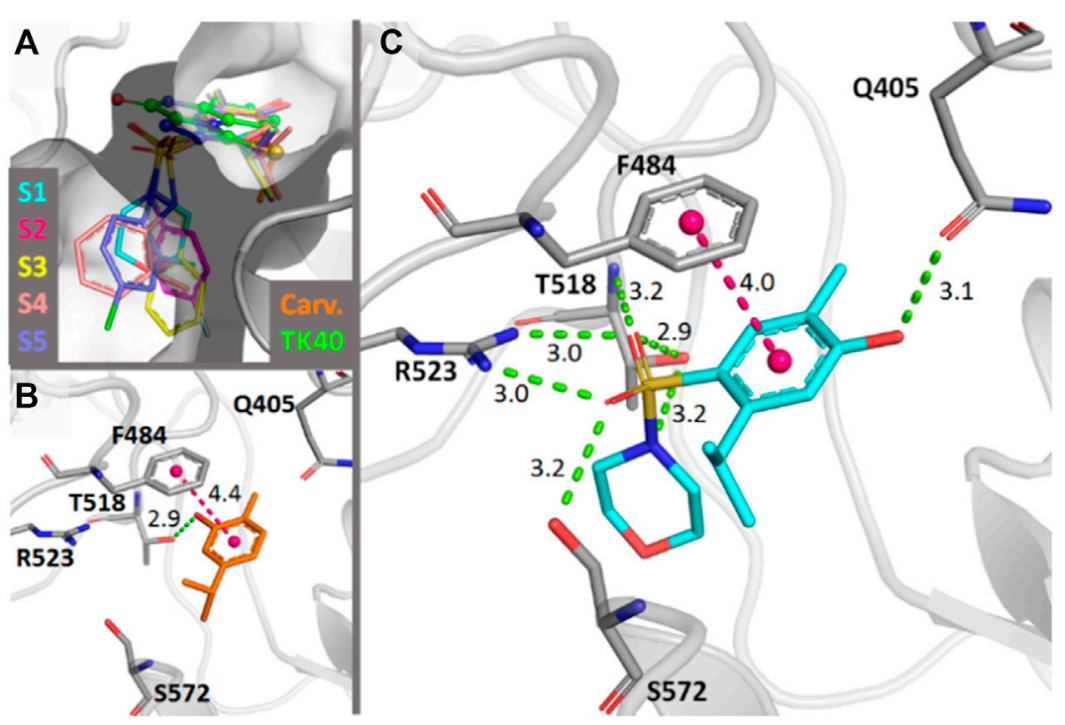

FIGURE 7 | (A) Docking-predicted binding modes in the rattus norvegicus NMDA-glycine binding site (PDB ID 4KFQ). The carbon atoms of each molecule are represented in a different color. The carbon atoms of the crystallographic antagonist TK40 are shown in green. (B) Main interactions established by carvacrol in the predicted binding mode. (C) Main interactions found by $\mathbf{S} \mathbf{1}$ in the predicted binding mode. Hydrogen bonds are represented in green and $\pi$-interactions in magenta. Distances are in $\AA$.

FIGURE 8 | Conformation of the crystallographic ligand in the binding site of NO synthase (PDB ID 6NGJ) after the redocking studies.

Extensive research has revealed that NO plays an essential role in several biological processes, such as neurotransmission, immune defense, and regulation of cell death (Snider and McMahon, 1998). The early 20th century witnessed the discovery of the role played by NO in nociception in both the central and peripheral levels (Zhuo and Gebhart, 1997). One of the physiological functions of NO was initially found in the vasculature; it was shown that the role of endothelium-derived relaxation factor (EDRF) could be quantitatively explained by the formation of NO by endothelial cells (Moncada and Higgs, 2006).

Treatment of pain with NO donors began with the use of nitroglycerin (NTG), which figures among the oldest treatments for ischemic heart disease (Boden et al., 2015). Discovered in
1847, NTG was used for the therapy of pain in angina pectoris for 100 years. However, its mechanism of action was not revealed until EDRF was identified as NO (Marsh and Marsh, 2000). Independently, $\mathrm{NO}$ was found to be an endogenous activator of soluble guanylate cyclase, resulting in the formation of cyclic GMP (cGMP), which acts as a second messenger in many cells, including the sensory neurons (Pereira et al., 2011).

$\mathrm{NO}$ is a highly reactive chemical messenger diffusible through the cytoplasmic membranes that is critical for the control of neuronal transmission, inflammation, cytotoxicity, and neural plasticity (Pacher et al., 2007). NO modulates the excitability of spinal sensory neurons and contributes to pain in different ways. The control of NO biosynthesis is regulated by NO synthase 

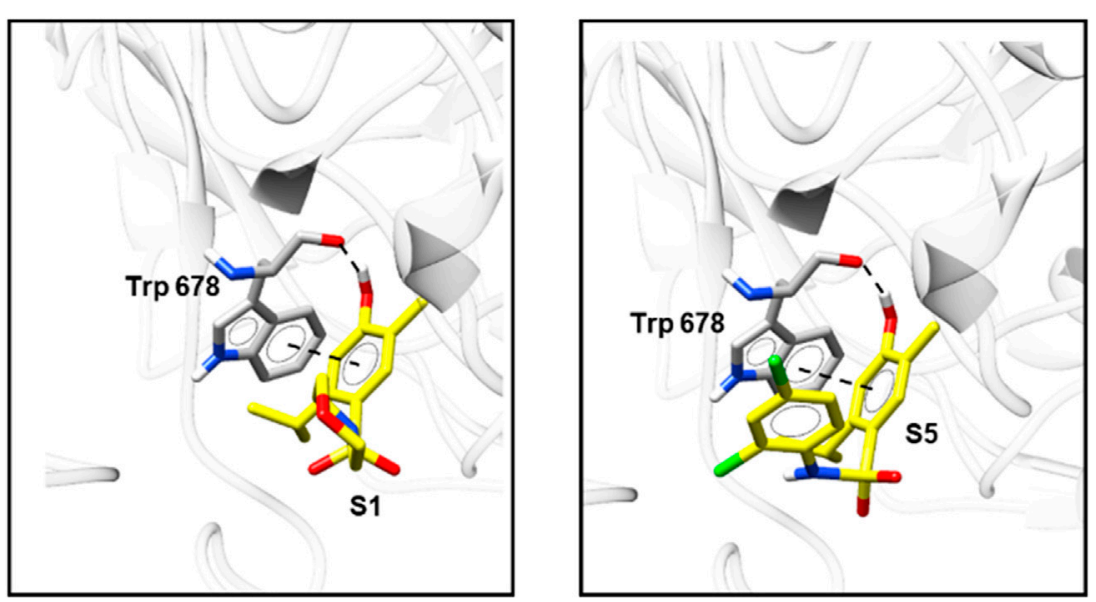

FIGURE $\mathbf{9}$ | Top-scoring docking poses for $\mathbf{S} \mathbf{1}$ and $\mathbf{S 5}$ in the binding site of NO synthase (PDB ID 6NGJ).

TABLE 2 | Molecular properties of sulfonamides S1-S5.
Property

S1

miLogP

TPSA $\left(\AA^{2}\right)$

Natoms

MW

HBA

HBD

nviolations

NRB

Molecular volume $\left(\AA^{3}\right)$

$\mathbf{S 2}$
4.20
66.40
22
323.39
4
2
0
4
278.75

S3

2.57

$$
79.29
$$

22

$$
320.41
$$$$
5
$$

2

0

5

286.46

$\begin{array}{cc}\mathbf{5 4} & \mathbf{S 5} \\ 3.77 & 5.32 \\ 86.62 & 66.40 \\ 22 & 23\end{array}$

$321.40 \quad 374.29$

54

32

$0 \quad 1$

$281.83 \quad 300.89$
(NOS) enzymes. Three NO synthase isoforms (NOS; EC 1.14.13.39) catalyze the production of NO (Förstermann and Sessa, 2012). They use $\mathrm{O}_{2}$ and $\mathrm{L}$-arginine as substrates and flavin mononucleotide (FMN), flavin adenine dinucleotide (FAD), reduced nicotinamide-adenine-dinucleotide phosphate
(NADPH), and tetrahydrobiopterin (BH4) as cofactors (Förstermann and Sessa, 2012). In this work, molecular docking was used to investigate NOS inhibition by the carvacrol derivatives.

\section{Quantum Studies}

The HOMO profile showed a variation of the charge density among the carvacrol derivatives. The HOMO and LUMO energies of compound S1 is -7.40 and $-0.62 \mathrm{eV}$, respectively. The electronic density is concentrated in the phenol group for HOMO and LUMO. Compound S2, however, differs regarding the position of the charge density for these orbitals. In HOMO $(-6.86 \mathrm{eV})$, the orbitals are concentrated on the fluoro-phenyl group. This is because fluorine tends to attract electron density (electronegative atom). In LUMO $(-0.75 \mathrm{eV})$, the electronic density tends to be favorable in the phenol group. The band gap in this compound is $-6.11 \mathrm{eV}$. Compound $\mathbf{S 3}$ has HOMO and LUMO energies of -7.35 and $-0.86 \mathrm{eV}$, respectively. The electronic density of HOMO tends to be located at the phenol. In

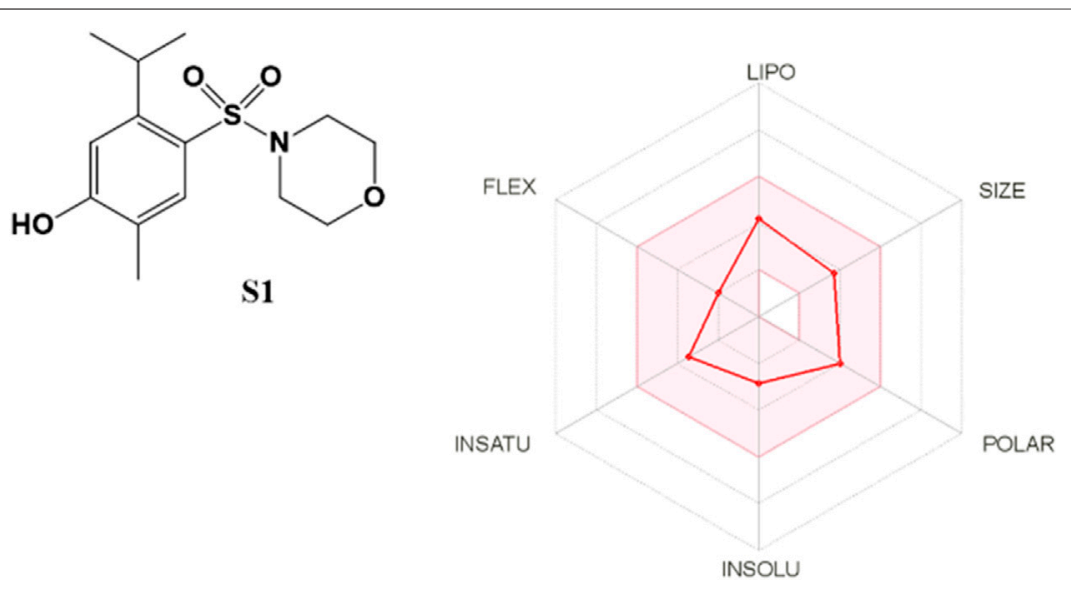

FIGURE 10 | The Bioavailability Radar for S1. The figure was generated online using SwissADME. Compound $\mathbf{S 1}$ combines good hydrophobicity and solubility, which is vital for membrane transport and permeability. Also, it does not violate any of the filters proposed by Lipinski, Ghose, Veber, Egan, and Muegge (Figure 11). 


\begin{tabular}{|ll|ll|}
\hline \multicolumn{2}{|c|}{ Lipophilicity } & \multicolumn{2}{c|}{ Druglikeness } \\
\hline Log $\mathrm{P}_{\mathrm{o} / \mathrm{w}}$ (iLOGP) & 2.40 & Lipinski & Yes; 0 violation \\
Log $\mathrm{P}_{\mathrm{o} / \mathrm{w}}$ (XLOGP3) & 1.83 & Ghose & Yes \\
$\log \mathrm{P}_{\mathrm{o} / \mathrm{w}}$ (WLOGP) & 2.54 & Veber & Yes \\
\hline $\log \mathrm{P}_{\mathrm{o} / \mathrm{w}}$ (MLOGP) & 0.89 & Egan & Yes \\
Log $\mathrm{P}_{\mathrm{o} / \mathrm{w}}$ (SILICOS-IT) & 1.75 & Muegge & Yes \\
Consensus Log $\mathrm{P}_{\mathrm{o} / \mathrm{w}}$ & 1.88 & Bioavailability Score & 0.55 \\
& & &
\end{tabular}

FIGURE 11 | Lipophilicity and drug likeness for $\mathbf{S 1}$. These parameters were generated online using SwissADME.

LUMO, however, the electronic density concentrates in the region of the pyridinic group. In compound S4, the HOMO charge density surrounds the phenolic substituent $(-6.83 \mathrm{eV})$. In LUMO, however, the charge density concentrates in the carvacrol fragment $(-0.59 \mathrm{eV})$. Differently from the other compounds, the charge distribution in S5 distributes throughout the structure in HOMO $(-7.38 \mathrm{eV})$ and LUMO $(-1.01 \mathrm{eV})$. In HOMO, the positive density concentrates on the sulfonamide group and $p$-fluorine atom. In LUMO, however, the same region is predominantly negative throughout the structure. In ascorbic acid, the dihydroxyfuran has the HOMO electron density $(-6.91 \mathrm{eV})$ close to the hydroxyl groups in the resonant region. In LUMO $(-0.49 \mathrm{eV})$, the signal of electronic density changes and concentrates close to the oxygen atom of the furan group.

\section{Antinociceptive Activity}

Injection of glutamate (i.pl.) in the mouse paw causes significant paw edema and nociception (Beirith et al., 2002; Meotti et al., 2010). Figure 4 shows that systemic administration of carvacrol, S1, S2, S3, and S5 significantly inhibits nociception induced by injection of $20 \mu \mathrm{mol} / \mathrm{paw}$ glutamate, suggesting that these compounds have an important therapeutic effect for the treatment of acute pain of inflammatory origin, probably due to a decrease in peripheral glutamatergic signaling. Treatment with the compounds significantly reduced pain behavior induced by glutamate (i.pl.), characterized by spontaneous licking/biting of the injected hind paw. Carvacrol was able to reduce nociceptive behavior by $19 \pm 6$ and $44 \pm 8 \%$ at 0.0003 and $0.003 \mathrm{mg} / \mathrm{kg}$, respectively. Moreover, the sulfonamides derived from carvacrol showed the following percent inhibitions: $16 \pm$ $5 \%, 62 \pm 5 \%$, and $50 \pm 7 \%$ for S1 at $0.0003,0.003$, and $0.03 \mathrm{mg} / \mathrm{kg}$, respectively; $26 \pm 5 \%$ and $34 \pm 6 \%$ for S2 at 0.003 and $0.03 \mathrm{mg} / \mathrm{kg}$, respectively; $25 \pm 9 \%$ and $22 \pm 5 \%$ for S3 at 0.003 and $0.03 \mathrm{mg} / \mathrm{kg}$, respectively; $39 \pm 8 \%$ and $27 \pm 13 \%$ for $\mathbf{S 5}$ at 0.003 and $0.03 \mathrm{mg} / \mathrm{kg}$, respectively.

The calculated mean $\mathrm{ID}_{50}$ value for sulfonamides derived from carvacrol was $0.002(0.001-0.002) \mathrm{mg} / \mathrm{kg}$ for S1, 0.442 $(0.063-0.387) \mathrm{mg} / \mathrm{kg}$. Thus, the results of the present study demonstrate that carvacrol and S1, S2, S3, and S5 reduce nociception induced by glutamate (i.pl.), suggesting that inhibition of the stimulatory mechanism via peripheral

\begin{tabular}{|c|c|c|c|}
\hline \multicolumn{2}{|c|}{ Medicinal Chemistry } & \multicolumn{2}{|c|}{ Pharmacokinetics } \\
\hline PAINS & 0 alert & Gl absorption & High \\
\hline Brenk & 0 alert & BBB permeant & Yes \\
\hline Leadlikeness & Yes & P-gp substrate & No \\
\hline Synthetic accessibility & 2.77 & CYP1A2 inhibitor & No \\
\hline & & CYP2C19 inhibitor & Yes \\
\hline & & CYP2C9 inhibitor & No \\
\hline & & CYP2D6 inhibitor & No \\
\hline & & CYP3A4 inhibitor & No \\
\hline $\mathrm{HO}$ & & $\begin{array}{l}\log K_{\mathrm{p}} \text { (skin } \\
\text { permeation) }\end{array}$ & $\begin{array}{l}-6.83 \\
\mathrm{~cm} / \mathrm{s}\end{array}$ \\
\hline \multicolumn{4}{|c|}{$\begin{array}{l}\text { FIGURE } 12 \text { | Medicinal Chemistry and pharmacokinetics for S1. These } \\
\text { parameters were generated online using SwissADME. }\end{array}$} \\
\hline
\end{tabular}

glutamatergic neurotransmission may contribute, at least in part, to the antinociceptive effect of these compounds. In addition, we would like to highlight that carvacrol and compounds S1, S2, S3, and S5 may be interesting lead compounds for acute pain, especially $\mathbf{S} 1(0.003 \mathrm{mg} / \mathrm{kg})$ since it presented the highest efficacy among the analyzed compounds.

Importantly, the compounds derived from carvacrol, selected to carry out the in vivo experiments, were chosen from the results presented in the molecular docking, quantum studies, and the in vitro antioxidant activity. Our results corroborate previous results (Arigesavan and Sudhandiran, 2015) which also found antioxidant and anti-inflammatory effects after treatment with carvacrol, using a carcinogenicity model in the colon of rats. Moreover, previous studies demonstrated that carvacrol attenuates mechanical hypernociception induced by carrageenan (Guimarães et al., 2012) and the acute pain acetic acid-induced abdominal constriction and formalin (Cavalcante Melo et al., 2012). Also, it was shown (Barnwal et al., 2018) that carvacrol increased the activities of antioxidant enzymes and downregulated expression by reducing the inflammation marker in positively dyed cells (iNOS, NF- $\kappa \mathrm{B}$, and COX-2) in a pulmonary toxicity model. These data from the literature reinforce the antinociceptive, anti-inflammatory, and antioxidant potential of carvacrol observed in our study.

Findings from the literature (Pacher et al., 2007; Förstermann and Sessa, 2012) indicate that superoxide $\left(\mathrm{SO}, \mathrm{O}^{(2) \bullet(-)}\right)$ and peroxynitrite $\left(\mathrm{PN}, \mathrm{ONOO}^{(-)}\right.$, the product of its reaction) are essential for the emergence of pain caused by different etiologies. These findings reinforce the concept that ROS play an essential part in NMDA activation, which is a critical ionotropic glutamatergic receptor, which contributes to central and peripheral pain. Therefore, this study supports previous results (Wang et al., 2004) that stated that superoxide mediates hyperalgesia (increased sensitivity to painful stimulation) through M40403, a manganese(II) complex with a bis(cyclohexylpyridine-substituted) macrocyclic ligand, which is a superoxide dismutase mimetic. These findings disclosed the central role played by superoxide in the peripheral signaling of nociception. In addition, it was shown that the M40403 antihyperalgesic activity could not be reverted by naloxone, which excludes the participation of opioid signaling cascades. 
Moreover, so far, few studies have investigated the effect of carvacrol on neurotransmitter modulation. The studies by Zotti et al. (2013) demonstrated that carvacrol, when ingested regularly in low concentrations, influences brain activity by increasing the levels of neurotransmitters such as serotonin and dopamine, which can determine feelings of well-being and reinforcing positive effects. Thus, our interest in investigating the glutamatergic system has arisen, considering that glutamate is a major mediator in the CNS, mediating excitatory neurotransmission in mammals, including in sensory neurons that convey pain, being strongly involved in the stimulation of peripheral and central pain. Therefore, our findings are unprecedented and relevant as they demonstrate the inhibitory capacity of carvacrol on the peripheral glutamatergic pathway.

It was shown (Kuo et al., 2017) that carvacrol mitigated injury in tissues and inflammation derived from periodontitis induced by ligation. Besides that, carvacrol proved to attenuate inflammatory response induced by carrageenan, decreasing mouse paw edema (Guimarães et al., 2012). These data from the literature support the anti-inflammatory, antinociceptive and antiedematogenic effects of carvacrol observed in our study. Importantly, paw edema and pain induced by glutamate are essentially associated with non-NMDA ionotropic glutamate receptors and NO production, a vasodilator, and an important neurotransmitter (Beirith et al., 2002). When in excess, it may be involved in the production of oxidative lesions in proteins. These findings reinforce the importance of studying glutamate-induced paw edema and nociception, as well as the beneficial effects of carvacrol and its derivatives found in this study.

Our results agree with literature data which demonstrated that carvacrol had no effect on the spontaneous locomotion in mice (Cavalcante Melo et al., 2012; Guimarães et al., 2012). However, these studies used a curve of carvacrol doses ranging from 25 to $100 \mathrm{mg} / \mathrm{kg}$ in the open-field test and we are the first group to test a much lower dose curve for carvacrol (0.0003, 0.003, and $0.03 \mathrm{mg} / \mathrm{kg}$ ) in pain, edema, and spontaneous locomotion. In addition, Guimarães et al. (2012) demonstrated that carvacrol at a dose of $100 \mathrm{mg} / \mathrm{kg}$ reduced the animals' ambulation in the openfield test, $30 \mathrm{~min}$ after intraperitoneal administration, showing that this dose is not safe as it causes nonspecific effects on locomotor activity and should be excluded in future pain studies. It is already well described that some drugs can cause motor slowness (bradykinesia) or even act as a muscle relaxant, causing non-specific changes in the locomotor activity of animals (Cartmell et al., 1991). In addition, drugs like benzodiazepines and other anxiolytics decrease the exploratory behavior of animals (Hazim et al., 2014). In this regard, it was demonstrated (Coderre and van Empel, 1994) that many glutamate antagonists, primarily via ionotropic NMDA receptor, such as the receptor channel block MK-801, produce significant antinociceptive effects, but decrease exploratory behavior of animals. In contrast, our results demonstrate that the intragastric treatment with the tested compounds can induce a significant antinociceptive effect via inhibition of peripheral glutamate, without causing any detectable motor dysfunction. Thus, carvacrol and its derivatives S1, S2, S3, and S4 at doses up to $0.03 \mathrm{mg} / \mathrm{kg}$ have an attractive analgesic potential to treat acute pain without causing CNS sedation.

\section{Molecular Docking}

In general, no significant binding modes were obtained concerning poses matching the available structural criteria of known antagonists (Ramírez and Caballero, 2018). Only the docking simulations in the NMDA-GluN 1 glycine binding site $\left(\mathrm{LBD}-\mathrm{GluN}_{1}\right.$ ) excelled, which agrees with previous observations for selective ligands of this site, such as HA-966, "which barely interacts with other ionotropic glutamate receptors" (PlanellsCases et al., 2005).

Considering the docking results and the non-ataxic effects of the compounds at the administered doses, the compounds are likely to be partial agonists, instead of agonists of the NMDAGluN1 glycine binding site, such as rapastinel (Wood et al., 2008) (GLYX-13 or BV-102), (+)-HA-966 (Millan and Seguin, 1993) and the recently reported 1-amino-1-cyclobutanecarboxylic acid (Fung et al., 2019).

\section{Molecular Properties}

The Lipinski RO5 applies to compounds that are active after oral administration. The RO5 includes four physicochemical property ranges $(\log \mathrm{P} \leq 5, \mathrm{MW} \leq 500, \mathrm{HBD} \leq 5$ and $\mathrm{HBA} \leq$ 10) that are present in $90 \%$ of the drugs that are active after oral administration and have reached phase II clinical development (Barret, 2018). The sulfonamides investigated in this work are within the RO5 desirable range, except for the miLogP of sulfonamide S5 (miLogP = 5.32), which is slightly higher than expected.

TPSA correlates with a compound's ability to permeate biological membranes through passive transport. Medicinal chemists use TPSA as an important parameter to optimize drug permeation through membranes. Molecules having TPSA values higher than $140 \AA^{2}$ are likely to permeate poorly into cell membranes (Pajouhesh and Lenz, 2005). For molecules that are required to act in the $\mathrm{CNS}$, penetration into the blood-brain barrier is needed, which requires a TPSA lower than $90 \AA^{2}$ (Hitchcock and Pennington, 2006). All investigated sulfonamides are in accordance with these parameters. A molecule that has a higher number of rotatable bonds becomes more flexible and have a good binding affinity with the binding pocket. For a potential drug candidate, Veber proposed that NRB should be $\leq 10$. All investigated sulfonamides are following this parameter.

The molecular volume assesses the transport properties of molecules such as blood-brain barrier penetration. The calculated values for this property are in line with the values expected for drug candidates.

During the discovery of novel drugs, molecules with useful therapeutic properties and low levels of toxicity are highly desirable. In this process, knowledge of the absorption, distribution, metabolism, and excretion profiles (ADME) is essential. It is well-known that the early evaluation of ADME during the drug discovery process reduces the attrition rates during clinical development. 


\section{CONCLUSION}

In this study, we report the SAR for a series of carvacrolderived sulfonamides. The antioxidant and antinociceptive activities of compounds S1-S5 were investigated using in vitro and in vivo assays. All the sulfonamides showed antioxidant activity in the in vitro tests comparable to that of the control compound (ascorbic acid). The results gathered in the in vitro antioxidant tests were linearly compared to the binding energies of the HOMO frontier orbital $\left(r^{2}=0.87\right.$ and 0.88$)$ calculated by DFT. The results of this study demonstrate that carvacrol and its derivatives S1, S2, S3, and S5 were able to reduce nociception induced by glutamate (i.pl.). Moreover, these findings show that the intragastric treatment with the tested compounds can induce a significant antinociceptive effect via inhibition of glutamatergic peripheral system without causing any detectable motor dysfunction, and not affecting the locomotor activity of mice. Thus, carvacrol and compounds S1, S2, S3, and S5 at doses up to $0.03 \mathrm{mg} / \mathrm{kg}$ have an attractive analgesic potential to treat acute pain with no CNS sedation. Docking simulations highlighted the interactions between the compounds and the NMDAGluN $_{1}$ glycine binding site, which suggested that these molecules act as selective partial agonists. Besides, compounds S1-S5 exhibit physicochemical parameters and pharmacokinetics compatible with drug candidates. Overall, sulfonamides S1-S5 are suitable starting points for further molecular optimization.

\section{DATA AVAILABILITY STATEMENT}

The original contributions presented in the study are included in the article/Supplementary Material, further inquiries can be directed to the corresponding authors.

\section{REFERENCES}

Arigesavan, K., and Sudhandiran, G. (2015). Carvacrol Exhibits Anti-Oxidant and Anti-Inflammatory Effects against 1, 2-Dimethyl Hydrazine Plus Dextran Sodium Sulfate Induced Inflammation Associated Carcinogenicity in the Colon of Fischer 344 Rats. Biochem. Biophys. Res. Commun. 461, 314-320. doi:10.1016/j.bbrc.2015.04.030

Barnwal, P., Vafa, A., Afzal, S. M., Shahid, A., Hasan, S. K., Alpashree, et al. (2018). Benzo(a)pyrene Induces Lung Toxicity and Inflammation in Mice: Prevention by Carvacrol. Hum. Exp. Toxicol. 37, 752-761. doi:10.1177/0960327117735572

Barret, R. (2018). “Lipinski's Rule of Five," in Medicinal Chemistry: Fundamentals. Editor R. Barret (Elsevier), 97-100. doi:10.1016/b978-1-78548-288-5.50006-8

Beirith, A., Santos, A. R. S., and Calixto, J. B. (2002). Mechanisms Underlying the Nociception and Paw Oedema Caused by Injection of Glutamate into the Mouse Paw. Brain Res. 924, 219-228. doi:10.1016/s0006-8993(01)03240-1

Berman, B. M., and Bausell, B. R. (2000). The Use of Non-Pharmacological Therapies by Pain Specialists. Pain 85, 313-315. doi:10.1016/S0304-3959(00)00258-X

Berman, H. M., Battistuz, T., Bhat, T. N., Bluhm, W. F., Bourne, P. E., Burkhardt, K., et al. (2002). The Protein Data Bank. Acta Crystallogr. Sect. D Biol. Crystallogr. 58, 899-907. doi:10.1107/S0907444902003451

Biswas, S. K. (2016). Does the Interdependence Between Oxidative Stress and Inflammation Explain the Antioxidant Paradox? Oxid. Med. Cell. Longev. 2016, 5698931. doi:10.1155/2016/5698931

\section{ETHICS STATEMENT}

The animal study was reviewed and approved by the Ethics Committee of the Federal University of Santa Catarina-UFSC (protocol number PP00745).

\section{AUTHOR CONTRIBUTIONS}

AO: writing-original draft, review \& and editing, synthesis, in vitro antioxidant tests, quantum studies, molecular docking, molecular properties, supervision; LL: synthesis; RN: supervision; RAY: supervision; CN-M: writing-original draft, in vivo tests; AS: quantum studies; DP-S: writing_original draft, molecular docking; MD-R: writing_original draft, molecular docking; LF: writing-review \& editing, molecular modeling; AA: writing-review \& editing, supervision; AS: writing_review \& editing, supervision.

\section{FUNDING}

The National Council for Scientific and Technological Development $(\mathrm{CNPq})$, the Coordination for the Improvement of Higher Education Personnel (CAPES, funding code 001, and access to GOLD suite CSD-System Software through Dot.Lib Brazil), and the Sao Paulo Research Foundation (FAPESP, CIBFar grant 2013/07600-3), Brazil.

\section{SUPPLEMENTARY MATERIAL}

The Supplementary Material for this article can be found online at: https://www.frontiersin.org/articles/10.3389/fphar.2021.788850/ full\#supplementary-material

Boden, W. E., Padala, S. K., Cabral, K. P., Buschmann, I. R., and Sidhu, M. S. (2015). Role of Short-Acting Nitroglycerin in the Management of Ischemic Heart Disease. Drug Des. Devel. Ther. 9, 4793-4805. doi:10.2147/DDDT.S79116

Burgess, G., and Williams, D. (2010). The Discovery and Development of Analgesics: New Mechanisms, New Modalities. J. Clin. Invest. 120, 3753-3759. doi:10.1172/JCI43195

Cartmell, S. M., Gelgor, L., and Mitchell, D. (1991). A Revised Rotarod Procedure for Measuring the Effect of Antinociceptive Drugs on Motor Function in the Rat. J. Pharmacol. Methods 26, 149-159. doi:10.1016/0160-5402(91)90063-b

Cavalcante Melo, F. H., Rios, E. R. V., Rocha, N. F. M., Citõ, M. D. C. D. O., Fernandes, M. L., and De Sousa, D. P. (2012). Antinociceptive Activity of Carvacrol (5-Isopropyl-2-Methylphenol) in Mice. J. Pharm. Pharmacol. 64, 1722-1729. doi:10.1111/j.2042-7158.2012.01552.x

Coderre, T. J., and Van Empel, I. (1994). The Utility of Excitatory Amino Acid (EAA) Antagonists as Analgesic Agents. II. Assessment of the Antinociceptive Activity of Combinations of Competitive and Non-Competitive NMDA Antagonists with Agents Acting at Allosteric-Glycine and Polyamine Receptor. Pain 59, 353-359. doi:10.1016/0304-3959(94)90021-3

Dantas, B. P. V., Alves, Q. L., de Assis, K. S., Ribeiro, T. P., and de Almeida, Mô. M. (2015). Participation of the TRP Channel in the Cardiovascular Effects Induced by Carvacrol in Normotensive Rat. Vascul. Pharmacol. 67-69, 48-58. doi:10.1016/j.vph.2015.02.016

de Oliveira, A. S., Llanes, L. C., Brighente, I. M. C., Nunes, R. J., Yunes, R. A., Máximo Junior, N., et al. (2016). New Sulfonamides Derived from Carvacrol: 
Compounds with High Antibacterial Activity against Resistant Staphylococcus aureus Strains. J. Biosci. Med. 4, 105-114. doi:10.4236/jbm.2016.47011

de Souza, M. M., Andreolla, M. C., Ribeiro, T. C., Gonçalves, A. E., Medeiros, A. R., de Souza, A. S., et al. (2020). Structure-Activity Relationships of Sulfonamides Derived from Carvacrol and Their Potential for the Treatment of Alzheimer's Disease. RSC Med. Chem. 11, 307-316. doi:10.1039/d0md00009d

Di Meo, S., Reed, T. T., Venditti, P., and Victor, V. M. (2016). Role of ROS and RNS Sources in Physiological and Pathological Conditions. Oxid. Med. Cell. Longev. 2016, 1245049. doi:10.1155/2016/1245049

Dickenson, A. H. (1995). Spinal Cord Pharmacology of Pain. Br. J. Anaesth. 75, 193-200. doi:10.1093/bja/75.2.193

Do, H. T., Li, H., Chreifi, G., Poulos, T. L., and Silverman, R. B. (2019). Optimization of Blood-Brain Barrier Permeability with Potent and Selective Human Neuronal Nitric Oxide Synthase Inhibitors Having a 2-Aminopyridine Scaffold. J. Med. Chem. 62, 2690-2707. doi:10.1021/acs.jmedchem.8b02032

Förstermann, U., and Sessa, W. C. (2012). Nitric Oxide Synthases: Regulation and Function. Eur. Heart J. 33 (7), 829-837. doi:10.1093/eurheartj/ehr304

Fundytus, M. E. (2001). Glutamate Receptors and Nociception: Implications for the Drug Treatment of Pain. CNS Drugs 15, 29-58. doi:10.2165/00023210200115010-00004

Fung, T., Asiri, Y. I., Taheri, K., Wall, R., Schwarz, S. K. W., Puil, E., et al. (2019). Antinociception by Intrathecal Delivery of the Novel Non-Opioid 1-Amino-1Cyclobutanecarboxylic Acid. Eur. J. Pain 23, 260-271. doi:10.1002/ejp.1301

Gordh, T., Karlsten, R., and Kristensen, J. (1995). Intervention with Spinal NMDA, Adenosine, and NO Systems for Pain Modulation. Ann. Med. 27, 229-234. doi:10.3109/07853899509031964

Guimarães, A. G., Xavier, M. A., de Santana, M. T., Camargo, E. A., Santos, C. A., and Brito, F. A. (2012). Carvacrol Attenuates Mechanical Hypernociception and Inflammatory Response. Naunyn Schmiedebergs Arch. Pharmacol. 385, 253-263. doi:10.1007/s00210-011-0715-x

Guindon, J., Walczak, J. S., and Beaulieu, P. (2007). Recent Advances in the Pharmacological Management of Pain. Drugs 67, 2121-2133. doi:10.2165/ 00003495-200767150-00002

Halgren, T. A. (1996). Merck Molecular Force Field. I. Basis, Form, Scope, Parameterization, and Performance of MMFF94. J. Comput. 17, 490-519. doi:10.1002/(sici) 1096-987x(199604)17:5/6<490:aid-jcc1>3.0.co;2-p

Hanwell, M. D., Curtis, D. E., Lonie, D. C., Vandermeerschd, T., Zurek, E., and Hutchison, G. R. (2012). Avogadro: An Advanced Semantic Chemical Editor, Visualization, and Analysis Platform. J. Cheminform. 4, 17. doi:10.1186/17582946-4-17

Hazim, A. I., Ramanathan, S., Parthasarathy, S., Muzaimi, M., and Mansor, S. M. (2014). Anxiolytic-like Effects of Mitragynine in the Open-Field and Elevated Plus-Maze Tests in Rats. J. Physiol. Sci. 64, 161-169. doi:10.1007/s12576-0140304-0

Hitchcock, S. A., and Pennington, L. D. (2006). Structure-Brain Exposure Relationships. J. Med. Chem. 49, 7559-7583. doi:10.1021/jm060642i

Hückel, E. (1931). Quantentheoretische Beiträge Zum Benzolproblem - I. Die Elektronenkonfiguration Des Benzols Und Verwandter Verbindungen. Z. für Phys. 70, 204-286. doi:10.1007/BF01339530

Jensen, T. S., Gottrup, H., Kasch, H., Nikolajsen, L., Terkelsen, A. J., and Witting, N. (2001). Has Basic Research Contributed to Chronic Pain Treatment? Acta Anaesthesiol. Scand. 45, 1128-1135. doi:10.1034/j.1399-6576.2001.450913.x

Koehn, F. E., and Carter, G. T. (2005). The Evolving Role of Natural Products in Drug Discovery. Nat. Rev. Drug Discov. 4, 206-220. doi:10.1038/nrd1657

Korb, O., Stützle, T., and Exner, T. E. (2009). Empirical Scoring Functions for Advanced Protein-Ligand Docking with PLANTS. J. Chem. Inf. Model. 49, 84-96. doi:10.1021/ci800298z

Kuo, P. J., Hung, T. F., Lin, C. Y., Hsiao, H. Y., Fu, M. W., and Hong, P. (2017). Carvacrol Ameliorates Ligation-Induced Periodontitis in Rats. J. Periodontol. 88, e120-e128. doi:10.1902/jop.2017.160618

Ling, C. C., Lui, L. Y., and So, W. K. (2012). Do Educational Interventions Improve Cancer Patients' Quality of Life and Reduce Pain Intensity? Quantitative Systematic Review. J. Adv. Nurs. 68, 511-520. doi:10.1111/j.13652648.2011.05841.x

Loeser, J. D., and Treede, R. D. (2008). The Kyoto Protocol of IASP Basic Pain Terminology. Pain 137, 473-477. doi:10.1016/j.pain.2008.04.025

Maioli, N. A., Zarpelon, A. C., Mizokami, S. S., Calixto-Campos, C., Guazelli, C. F. S., and Hohmann, M. S. N. (2015). The Superoxide Anion Donor, Potassium
Superoxide, Induces Pain and Inflammation in Mice through Production of Reactive Oxygen Species and Cyclooxygenase-2. Braz. J. Med. Biol. Res. 48, 321-331. doi:10.1590/1414-431X20144187

Manchope, M. F., Calixto-Campos, C., Coelho-Silva, L., Zarpelon, A. C., PinhoRibeiro, F. A., and Georgetti, S. R. (2016). Naringenin Inhibits Superoxide Anion-Induced Inflammatory Pain: Role of Oxidative Stress, Cytokines, Nrf-2 and the No-CGMP-PKG-KATP Channel Signaling Pathway. PLoS One 11, e0153015. doi:10.1371/journal.pone. 0153015

Marsh, N., and Marsh, A. (2000). A Short History of Nitroglycerine and Nitric Oxide in Pharmacology and Physiology. Clin. Exp. Pharmacol. Physiol. 27, 313-319. doi:10.1046/j.1440-1681.2000.03240.x

Melo, F. H. C., Venâncio, E. T., De Sousa, D. P., de Franca Fonteles, M. M., De Vasconcelos, S. M. M., Viana, G. S. B., et al. (2010). Anxiolytic-like Effect of Carvacrol (5-Isopropyl-2-Methylphenol) in Mice: Involvement with GABAergic Transmission. Fundam. Clin. Pharmacol. 24, 437-443. doi:10.1111/j.1472-8206.2009.00788.x

Meotti, F. C., Coelho Idos, S., and Santos, A. R. (2010). The Nociception Induced by Glutamate in Mice is Potentiated by Protons Released into the Solution. J. Pain 11, 570-578. doi:10.1016/j.jpain.2009.09.012

Millan, M. J., and Seguin, L. (1993). (+)-HA 966, a Partial Agonist at the Glycine Site Coupled to NMDA Receptors, Blocks Formalin-Induced Pain in Mice. Eur. J. Pharmacol. 238, 445-447. doi:10.1016/0014-2999(93)90884-k

Moncada, S., and Higgs, E. A. (2006). The Discovery of Nitric Oxide and Its Role in Vascular Biology. Br. J. Pharmacol. 147 (Suppl. 1), S193-S201. doi:10.1038/ sj.bjp. 0706458

Moncada, S., Rees, D. D., Schulz, R., and Palmer, R. M. J. (1991). Development and Mechanism of a Specific Supersensitivity to Nitrovasodilators after Inhibition of Vascular Nitric Oxide Synthesis In Vivo. Proc. Natl. Acad. Sci. U. S. A. 88, 2166-2170. doi:10.1073/pnas.88.6.2166

Nageswari, G., George, G., Ramalingam, S., and Govindarajan, M. (2018). Electronic and Vibrational Spectroscopic (FT-IR and FT-Raman) Investigation Using Ab Initio (HF) and DFT (B3LYP and B3PW91) and HOMO/LUMO/MEP Analysis on the Structure of L-Serine Methyl Ester Hydrogen Chloride. J. Mol. Struct. 1166, 422-441. doi:10.1016/j.molstruc.2018.04.014

Nucci-Martins, C., Nascimento, L. F., Venzke, D., Brethanha, L. C., Sako, A. V. F, Oliveira, A. S., et al. (2016). Antinociceptive Effect of Hydroalcoholic Extract and Isoflavone Isolated from Polygala Molluginifolia in Mice: Evidence for the Involvement of Opioid Receptors and TRPV1 and TRPA1 Channels. Phytomedicine 23, 429-440. doi:10.1016/j.phymed.2016.02.002

Oliveira, A. S. de., de Souza, L. F. S., Nunes, R. J., Johann, S., Palomino-Salcedo, D. L., Ferreira, L. L. G., et al. (2020). Antioxidant and Antibacterial Activity of Sulfonamides Derived from Carvacrol: A Structure-Activity Relationship Study. Curr. Top. Med. Chem. 20, 173-181. doi:10.2174/1568026619666191127144336

Pacher, P., Beckman, J. S., and Liaudet, L. (2007). Nitric Oxide and Peroxynitrite in Health and Disease. Physiol. Rev. 87, 315-424. doi:10.1152/physrev.00029.2006

Pajouhesh, H., and Lenz, G. R. (2005). Medicinal Chemical Properties of Successful Central Nervous System Drugs. NeuroRx 2, 541-553. doi:10.1602/ neurorx.2.4.541

Pereira, A. C., Paulo, M., Araújo, A. V., Rodrigues, G. J., and Bendhack, L. M. (2011). Nitric Oxide Synthesis and Biological Functions of Nitric Oxide Released from Ruthenium Compounds. Braz. J. Med. Biol. Res. 44, 947-957. doi:10.1590/s0100-879x2011007500084

Phaniendra, A., Jestadi, D. B., and Periyasamy, L. (2015). Free Radicals: Properties, Sources, Targets, and Their Implication in Various Diseases. Indian J. Clin. Biochem. 30, 11-26. doi:10.1007/s12291-014-0446-0

Pires, P. W., Sullivan, M. N., Pritchard, H. A. T., Robinson, J. J., and Earley, S. (2015). Unitary TRPV3 Channel Ca 2+ Influx Events Elicit EndotheliumDependent Dilation of Cerebral Parenchymal Arterioles. Am. J. Physiol. Circ. Physiol. 309, H2031-H2041. doi:10.1152/ajpheart.00140.2015

Planells-Cases, R., Perez-Paya, E., Messeguer, A., Carreno, C., and Ferrer-Montiel, A. (2003). Small Molecules Targeting the NMDA Receptor Complex as Drugs for Neuropathic Pain. Mini Rev. Med. Chem. 3, 749-756. doi:10.2174/ 1389557033487782

Ramírez, D., and Caballero, J. (2018). Is it Reliable to Take the Molecular Docking Top Scoring Position as the Best Solution without Considering Available Structural Data? Molecules 23, 1038. doi:10.3390/molecules23051038

Rao, K. S. (2009). Free Radical Induced Oxidative Damage to DNA: Relation to Brain Aging and Neurological Disorders. Indian J. Biochem. Biophys. 46, 9-15. 
Ravn, P., Madhurantakam, C., Kunze, S., Matthews, E., Priest, C., O’Brien, S., et al. (2013). Structural and Pharmacological Characterization of Novel Potent and Selective Monoclonal Antibody Antagonists of Glucose-Dependent Insulinotropic Polypeptide Receptor. J. Biol. Chem. 288, 19760-19772. doi:10.1074/jbc.M112.426288

Rodrigues, T., Reker, D., Schneider, P., and Schneider, G. (2016). Counting on Natural Products for Drug Design. Nat. Chem. 8, 531-541. doi:10.1038/ nchem.2479

Rowlingson, J. C. (2000). Textbook of Pain. Anesth. Analg. 91, 1315. doi:10.1213/ 00000539-200011000-00066

Salvemini, D., Little, J. W., Doyle, T., and Neumann, W. L. (2011). Roles of Reactive Oxygen and Nitrogen Species in Pain. Free Radic. Biol. Med. 51, 951-966. doi:10.1016/j.freeradbiomed.2011.01.026

Schmidt, B. L., Hamamoto, D. T., Simone, D. A., and Wilcox, G. L. (2010). Mechanism of Cancer Pain. Mol. Interv. 10, 164-178. doi:10.1124/mi.10.3.7

Schmidt, M. W., Baldridge, K. K., Boatz, J. A., Elbert, S. T., Gordon, M. S., Jensen, J., et al. (1993). General Atomic and Molecular Electronic Structure System. J. Comput. Chem. 14, 1347-1363. doi:10.1002/jcc.540141112

Sens, L., De Oliveira, A. S., Mascarello, A., Brighente, I. M. C., Yunes, R. A., and Nunes, R. J. (2018). Synthesis, Antioxidant Activity, Acetylcholinesterase Inhibition and Quantum Studies of Thiosemicarbazones. J. Braz. Chem. Soc. 29, 343-352. doi:10.21577/0103-5053.20170146

Silva, J. C., Raquel, S., Lima, G. De., Gonçalves, R., Júnior, D. O., and Guedes, R. (2013). Modelos Experimentais Para Avaliação da Atividade Antinociceptiva de Produtos Naturais : Uma Revisão. Rev. Bras. Farm. 94, 18-23.

Snider, W. D., and McMahon, S. B. (1998). Tackling Pain at the Source: New Ideas about Nociceptors. Neuron 20, 629-632. doi:10.1016/s0896-6273(00) 81003-x

Tominaga, M., Numazaki, M., Iida, T., and Tominaga, T. (2003). Molecular Mechanisms of Nociception. Nihon Shinkei Seishin Yakurigaku Zasshi 23, 139-147.

Veber, D. F., Johnson, S. R., Cheng, H.-Y., Smith, B. R., Ward, K. W., and Kopple, K. D. (2002). Molecular Properties That Influence the Oral Bioavailability of Drug Candidates. J. Med. Chem. 45, 2615-2623. doi:10.1021/jm020017n

Verri, W. A., Cunha, T. M., Parada, C. A., Poole, S., Cunha, F. Q., and Ferreira, S. H. (2006). Hypernociceptive Role of Cytokines and Chemokines: Targets for
Analgesic Drug Development? Pharmacol. Ther. 112, 116-138. doi:10.1016/ j.pharmthera.2006.04.001

Wang, Z. Q., Porreca, F., Cuzzocrea, S., Galen, K., Lightfoot, R., Masini, E., et al. (2004). A Newly Identified Role for Superoxide in Inflammatory Pain. J. Pharmacol. Exp. Ther. 309, 869-878. doi:10.1124/jpet.103.064154

Wood, P. L., Mahmood, S. A., and Moskal, J. R. (2008). Antinociceptive Action of GLYX-13: An N-Methyl-D-Aspartate Receptor Glycine Site Partial Agonist. Neuroreport 19, 1059-1061. doi:10.1097/WNR.0b013e32830435c9

Zhuo, M., and Gebhart, G. F. (1997). Biphasic Modulation of Spinal Nociceptive Transmission From the Medullary Raphe Nuclei in the Rat. J. Neurophysiol. 78, 746-758. doi:10.1152/jn.1997.78.2.746

Zimmermann, M. (1983). Ethical Guidelines for Investigations of Experimental Pain in Conscious Animals. Pain 16, 109-110. doi:10.1016/0304-3959(83) 90201-4

Zotti, M., Colaianna, M., Morgese, M. G., Tucci, P., Schiavone, S., Avato, P., et al. (2013). Carvacrol: From Ancient Flavoring to Neuromodulatory Agent. Molecules 18, 6161-6172. doi:10.3390/molecules18066161

Conflict of Interest: The authors declare that the research was conducted in the absence of any commercial or financial relationships that could be construed as a potential conflict of interest.

Publisher's Note: All claims expressed in this article are solely those of the authors and do not necessarily represent those of their affiliated organizations, or those of the publisher, the editors and the reviewers. Any product that may be evaluated in this article, or claim that may be made by its manufacturer, is not guaranteed or endorsed by the publisher.

Copyright (C) 2021 de Oliveira, Llanes, Nunes, Nucci-Martins, de Souza, PalominoSalcedo, Dávila-Rodríguez, Ferreira, Santos and Andricopulo. This is an open-access article distributed under the terms of the Creative Commons Attribution License (CC $B Y)$. The use, distribution or reproduction in other forums is permitted, provided the original author(s) and the copyright owner(s) are credited and that the original publication in this journal is cited, in accordance with accepted academic practice. No use, distribution or reproduction is permitted which does not comply with these terms. 